\title{
Identification of 35 C-Type Lectins in the Oriental Armyworm, Mythimna separata (Walker)
}

\author{
Hao Li ${ }^{1}$, Fang-Fang Liu ${ }^{1}$, Li-Qing Fu ${ }^{1}$, Ze Liu ${ }^{1}$, Wen-Ting Zhang ${ }^{1}$, Qian Wang ${ }^{1}$ and Xiang-Jun Rao ${ }^{1,2, *(D)}$ \\ 1 School of Plant Protection, Anhui Agricultural University, Hefei 230036, China; 19720142@ahau.edu.cn (H.L.); \\ 19720147@ahau.edu.cn (F.-F.L.); 18720054@ahau.edu.cn (L.-Q.F.); 1lzz@ahau.edu.cn (Z.L.); \\ 15103867@ahau.edu.cn (W.-T.Z.); 20720220@ahau.edu.cn (Q.W.) \\ 2 Anhui Province Key Laboratory of Integrated Pest Management on Crops, Anhui Agricultural University, \\ Hefei 230036, China \\ * Correspondence: rxj@ahau.edu.cn
}

check for updates

Citation: Li, H.; Liu, F.-F.; Fu, L.-Q.; Liu, Z.; Zhang, W.-T.; Wang, Q.; Rao, X.-J. Identification of 35 C-Type Lectins in the Oriental Armyworm, Mythimna separata (Walker). Insects 2021, 12, 559. https://doi.org/ $10.3390 /$ insects 12060559

Academic Editor: Peter Piermarini

Received: 28 May 2021

Accepted: 14 June 2021

Published: 16 June 2021

Publisher's Note: MDPI stays neutral with regard to jurisdictional claims in published maps and institutional affiliations.

Copyright: (c) 2021 by the authors. Licensee MDPI, Basel, Switzerland. This article is an open access article distributed under the terms and conditions of the Creative Commons Attribution (CC BY) license (https:// creativecommons.org/licenses/by/ $4.0 /)$.
Simple Summary: The oriental armyworm Mythimna separata is a lepidopteral agricultural pest that causes serious damage to many crops, such as maize, wheat, and sorghum. To control this pest, it is advisable to take comprehensive measures, including the use of chemical pesticides, microbial pesticides, and cultural practices. However, microbial pesticides (entomopathogens) can be eliminated by the insect immune system. C-type lectins (CTLs) are a family of pattern-recognition receptors that recognize carbohydrates and mediate immune responses. C-type lectins in the oriental armyworm have not yet been identified and characterized. In this study, a transcriptome of $M$. separata larvae was constructed and a total of 35 CTLs containing single or dual carbohydrate-recognition domains (CRDs) were identified from unigenes. Phylogenetic analyses, sequence alignments and structural predictions were performed. Gene expression profiles in different developmental stages, naïve larval tissues, and bacteria/fungi-challenged larvae were analyzed. Overall, our findings indicate that most dual-CRD CTLs are expressed in mid-late-stage larvae, pupae, and adults. Bacterial and fungal challenges can stimulate the expression of many CTLs in larval hemocytes, fat body, and midgut. Our data suggest the importance of CTLs in immune responses of M. separata.

Abstract: Insect C-type lectins (CTLs) play vital roles in modulating humoral and cellular immune responses. The oriental armyworm, Mythimna separata (Walker) (Lepidoptera: Noctuidae) is a migratory pest that causes significant economic loss in agriculture. CTLs have not yet been systematically identified in $M$. separata. In this study, we first constructed a transcriptome of $M$. separata larvae, generating a total of 45,888 unigenes with an average length of $910 \mathrm{bp}$. Unigenes were functionally annotated in six databases: NR, GO, KEGG, Pfam, eggNOG, and Swiss-Prot. Unigenes were enriched in functional pathways, such as those of signal transduction, endocrine system, cellular community, and immune system. Thirty-five unigenes encoding C-type lectins were identified, including CTLS1 CTL-S6 (single CRD) and IML-1 IML-29 (dual CRD). Phylogenetic analyses showed dramatic lineage-specific expansions of IMLs. Sequence alignment and structural modeling identified potential ligand-interacting residues. Real-time qPCR revealed that CTL-Ss mainly express in eggs and early stage larvae, while IMLs mainly express in mid-late-stage larvae, pupae, and adults. In naïve larvae, hemocytes, fat body, and epidermis are the major tissues that express CTLs. In larvae challenged by Escherichia coli, Staphylococcus aureus, or Beauveria bassiana, the expression of different CTLs was stimulated in hemocytes, fat body and midgut. The present study will help further explore functions of M. separata CTLs.

Keywords: Mythimna separata; C-type lectin; transcriptome

\section{Introduction}

Insects depend on the innate immune system to recognize and eliminate pathogens [1] Germline-encoded pattern recognition receptors (PRRs) can recognize pathogen-associated 
molecular patterns (PAMPs) to trigger immune responses. Common PAMPs include bacterial lipopolysaccharide, lipoteichoic acid, peptidoglycans, fungal glucans, viral capsid, nucleic acids, and parasitic molecular patterns [2]. Numerous insect PRRs have been identified, such as C-type lectins (CTLs), peptidoglycan-recognition proteins (PGRPs), and $\beta-1,3-$ glucan recognition proteins ( $\beta$ GRPs) [3-6].

CTLs are ubiquitous in plants, invertebrates, fungi, bacteria, and vertebrates. CTLs contain at least one carbohydrate-recognition domain (CRD) or C-type lectin-like domain (CTLD). Insect CTLs can be classified based on the domain architecture: CTL-S has a single CRD; immulectin (IML) has dual CRD; CTL-X has CRD and other motifs [7,8]. CTL-S and CTL-X are identified in many insect orders, while IMLs almost only exist in Lepidoptera [5]. Most CRDs consist of 110 130 amino acids and one to four $\mathrm{Ca}^{2+}$ binding sites. $\mathrm{Ca}^{2+}$ helps to maintain a stable protease-resistant structure. $\mathrm{Ca}^{2+}$ at site 2 mediates the recognition of carbohydrates through coordination bonds formed with hydroxyl groups on the sugar ring and amino acid side chains. CRDs with Glu-Pro-Asn (EPN) and GlnPro-Asp (QPD) motifs generally recognize mannose-type and galactose-type ligands, respectively. The differential arrangement of $3-\mathrm{OH}$ and $4-\mathrm{OH}$ on the pyranose ring is a major determinant in ligand preference [9]. In rat mannose-binding protein MBP-A, Glu ${ }^{193}$, $\mathrm{Asn}^{205}, \mathrm{Asp}^{206}, \mathrm{Glu}^{185}, \mathrm{Asn}^{187}, \mathrm{Ca}^{2+}$, and mannose form a ternary complex through a network of coordination and hydrogen bonds [10].

CTLs are involved in regulating humoral responses (phenoloxidase activation and antimicrobial peptide production) and cellular responses (encapsulation, nodulation, and phagocytosis). Manduca sexta immulectin-1 and immulectin-2 stimulate prophenol oxidase [11,12]. M. sexta immulectin-4 enhanced hemocyte encapsulation and melanization [13]. Two Drosophila CTLs can enhance encapsulation and melanization [14]. Knockdown of a CTL in Tribolium castaneum caused a significant decrease in antimicrobial peptides and transcription factors under lipopolysaccharide and peptidoglycan stimulation [15]. Helicoverpa armigera C-type lectin 7 can enhance hemocytes-mediated encapsulation and melanization [16]. H. armigera CTL14 depletion decreased the resistance to fungal challenge [17]. Bombyx mori lipopolysaccharide-binding protein participates in hemocyte nodule formation [18]. B. mori multibinding protein can trigger nodule reaction [19]. Some pathogens can subvert or use host CTLs to assist infection. The parasitoid, Pteromalus puparum, can suppress immune responses of the host, Pieris rapae, by silencing host CTL expression [20]. An Aedes aegypti C-type lectin (mosGCTL-1) facilitates West Nile Virus infection [21].

Caterpillars of the oriental armyworm feed on young seedlings or leaves of maize, wheat, sorghum, and millets. A large armyworm population may cause defoliation or damage corn cobs [22]. Using entomopathogens for pest control can efficiently prevent pesticide resistance [23]. Understanding the molecular interactions between entomopathogens and insects may help to develop new strategies for pest management [24]. Although a few immune factors in $M$. separata have been identified, less is known about its CTL family [25-27]. This study aims to construct a transcriptome of $M$. separata larvae and identify CTLs. In addition, the bioinformatic analyses and expression pattern assays will help to elucidate their roles in the development and immune system.

\section{Materials and Methods}

\subsection{Insects and Microorganisms}

Mythimna separata were collected in an experimental field at Anhui Agricultural University, Hefei, China. Larvae and adults were reared at $25^{\circ} \mathrm{C}$ (photoperiod 12L:12D). Microorganisms used in the study (Escherichia coli DH5 $\alpha$, Staphylococcus aureus, and Beauveria bassiana ARSEF 2860) were kindly given by Dr. Erjun Ling from the Institute of Plant Physiology and Ecology, Shanghai, China. 


\subsection{RNA Sample Preparation, Library Construction, and Sequencing}

Fourth instar larvae were frozen in liquid nitrogen and stored at $-80^{\circ} \mathrm{C}$. Total RNA was isolated using the Trizol Reagent (Invitrogen Life Technologies, Carlsbad, CA, USA). RNA concentration was determined using a NanoDrop spectrophotometer (Thermo Scientific, Waltham, MA, USA). RNA quality and integrity were determined by RNA agarose gel electrophoresis and Agilent Bioanalyzer 2100 system. Three micrograms of RNA were used as input material for the RNA sample preparations. Sequencing libraries were generated using the TruSeq RNA Sample Preparation Kit (Illumina, San Diego, CA, USA). To select cDNA fragments of the preferred $200 \mathrm{bp}$ in length, the library fragments were purified using the AMPure XP system (Beckman Coulter, Beverly, CA, USA). DNA fragments with ligated adaptor molecules on both ends were selectively enriched using Illumina PCR Primer Cocktail in a 15 cycle PCR reaction. Products were purified and quantified using the Agilent high sensitivity DNA assay on a Bioanalyzer 2100 system. The sequencing library was sequenced on a NovaSeq 6000 platform (Illumina) by Personal Biotechnology Co., Ltd (Nanjing, Jiangsu, China).

\subsection{De Novo Transcriptome Analysis Flow}

Raw data were filtered to remove low-quality reads using Cutadapt v2.7 to generate clean data ( $>10$ bp overlap: AGATCGGAAG; $20 \%$ base error rate was allowed) [28]. Trinity v2.5.1 with the default setting was used to montage clean reads to generate transcript sequence files [29]. The longest transcript of each gene (Unigene) was extracted as the representative sequence of the gene. Databases used in gene annotation include NR (NCBI non-redundant protein sequences), GO (Gene Ontology), KEGG (Kyoto Encyclopedia of Genes and Genome), eggNOG (evolutionary genealogy of genes: Non-supervised Orthologous Groups), Swiss-Prot, and Pfam.

\subsection{Identification of CTLs from the Transcriptome and Bioinformatic Analyses}

To identify unigenes that encode CTLs, other annotated CTLs (Manduca sexta, Bombyx mori, Spodoptera litura, Helicoverpa armigera, Apis mellifera) were used as queries for tblastn searches [30]. Putative CTL sequences were confirmed by searching the NCBI nonredundant protein database using blastx (cut-off E-value: 1e-100). Candidate sequences were amplified from larval cDNA by PCR and sequenced (Table S1). CRD regions were predicted by ScanProsite (https:/ / prosite.expasy.org/scanprosite/) (accessed on 10 May 2021). Sequences were aligned with MUSCLE and decorated in Jalview [31]. Phylogenetic trees were constructed in MEGA $X$ with the neighbor-joining method and visualized with Figtree v1.4.4 [32]. $\mathrm{Ca}^{2+}$ and sugar-binding sites were predicted by I-TASSER [33]. Molecular graphics were generated using Chimera v1.14 [34]. Sequence logos were generated with WebLogo [35]. Heatmaps were generated using TBtools [36].

\subsection{Analyses of the Expression Profiles by Real-Time qPCR (RT-qPCR)}

To explore the expression profile in different developmental stages, samples from six stages (eggs, early stage larvae, mid-stage larvae, late-stage larvae, pupa, and adults) were ground in liquid nitrogen and stored at $-80^{\circ} \mathrm{C}$. To explore the expression profile in naïve larval tissues, fifth instar larvae were anesthetized on ice and dissected to collect hemocytes, fat body, midgut, epidermis, and Malpighian tube. Collected tissues were immediately homogenized in SparkZol reagent (Sparkjade Biotechnology Co., Ltd., Jinan, Shandong, China). For the gene induction analysis, E. coli, S. aureus, and B. bassiana conidia were inactivated with $3 \%$ formaldehyde. Moreover, $10^{4}$ bacteria were resuspended in PBS (10 mM phosphate buffer, $37 \mathrm{mM} \mathrm{NaCl}, 2.7 \mathrm{mM} \mathrm{KCl}, \mathrm{pH} 7.4$ ) and injected into fifth instar larvae with a microsyringe. PBS was injected as the negative control. B. bassiana conidia were resuspended in PBS with $0.05 \%$ tween- 80 , and $4 \times 10^{4}$ conidia were injected into larvae. PBS with $0.05 \%$ tween- 80 was injected as the negative control. Hemocytes, fat body, and midgut were collected 6 and $24 \mathrm{~h}$ post-injection. Three larvae were used in each group, and all experiments were performed in three replicates. cDNA was synthesized from $1 \mu \mathrm{g}$ 
total RNA using SPARKscript II RT Plus Kit (with gDNA Eraser). RT-qPCR $\left(95{ }^{\circ} \mathrm{C} 30 \mathrm{~s}\right.$, 40 cycles of $95^{\circ} \mathrm{C} 5 \mathrm{~s}, 60^{\circ} \mathrm{C} 30 \mathrm{~s}$ ) was performed using MonAmp ${ }^{\mathrm{TM}}$ ChemoHS qPCR Mix (Monad Biotech Co., Ltd., Suzhou, Jiangsu, China) with the CFX96 real-time PCR detection system (Bio-Rad, Hercules, CA, USA). The relative gene expression level was calculated by the $2^{-\Delta \Delta C T}$ method. Primer information was provided in Table S1.

\section{Results}

3.1. Transcriptome Sequencing, Unigene Assembly, and Functional Annotation

A cDNA library was constructed for $M$. separata larvae and sequenced using the Illumina platform. This run produced 47,594,876 raw reads and 44,966,148 clean reads (Clean reads: 94.47\%, Q20: 97.45\%, Q30: 93.07\%). The clean reads were assembled into 81,837 transcripts with a mean length of $1135 \mathrm{bp}$. Transcripts were further assembled into 45,888 unigenes with a mean length of $910 \mathrm{bp}(\mathrm{N} 50=1687 \mathrm{bp})$. The transcriptome dataset was deposited in Sequence Read Archive (PRJNA702891).

Unigenes were annotated in six databases: NR, GO, KEGG, Pfam, eggNOG, and Swiss-Prot (Table 1). Approximately 18,157 unigenes were annotated to the NR database. The top 10 species with the most matches in the NR database were shown in Figure 1. In total, 4041 unigenes were matched to Amyelois transitella, followed by Bombyx mori (3679), Papilio xuthus (1673), Operophtera brumata (1324), Papilio machaon (1229), Danaus plexippus (1226), Plutella xylostella (970), Papilio polytes (931), Helicoverpa armigera (421), and Mythimna separata (142).

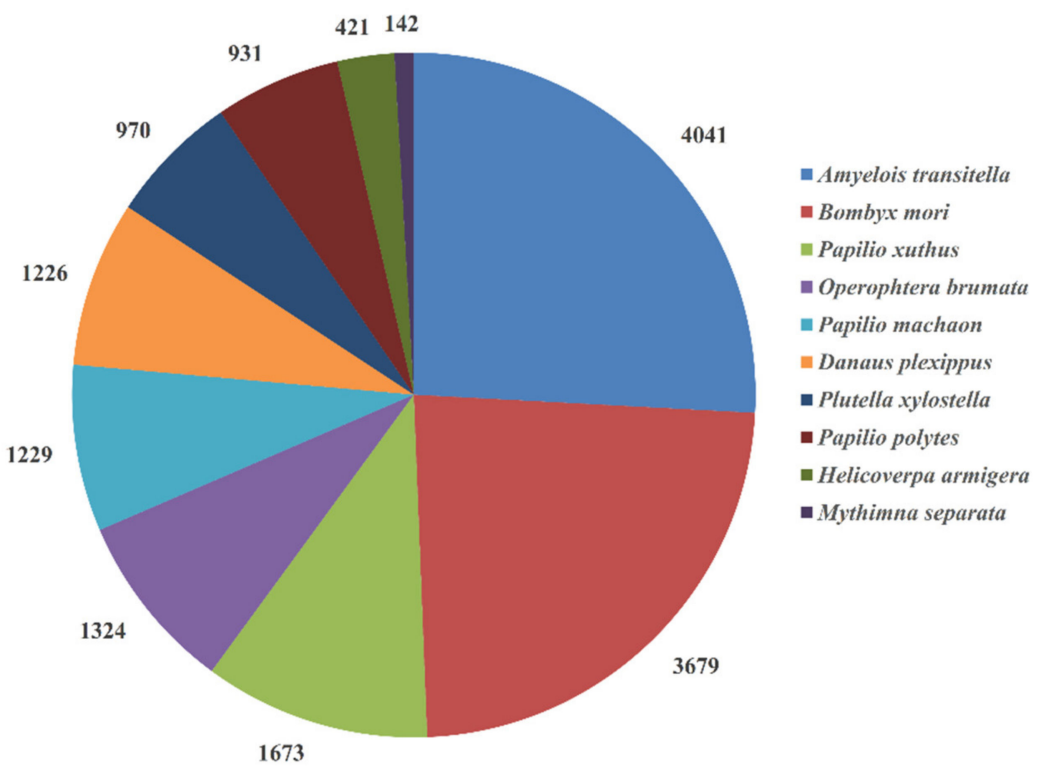

Figure 1. The top 10 species distribution in the NR database. Unigenes were aligned with the NCBI NR protein database. The top 10 species with numbers of matches were shown.

Table 1. Functional annotation of unigenes in databases.

\begin{tabular}{ccc}
\hline Database & Number & Percentage \\
\hline NR & 18,157 & 39.57 \\
GO & 8035 & 17.51 \\
KEGG & 8836 & 19.26 \\
Pfam & 9527 & 20.76 \\
eggNOG & 17,125 & 37.32 \\
Swiss-Prot & 11,142 & 24.28 \\
In all database & 3564 & 7.77 \\
\hline
\end{tabular}


Functions of unigenes were classified by GO assignments with BLAST2GO (Figure 2). A total of 36,622 unigenes were categorized into 55 categories, which belonged to three main categories: biological process (23), cellular component (19), and molecular function (13). The top 10 categories were binding (3584), cellular process (3569), catalytic activity (3564), metabolic process (3346), single-organism process (2640), membrane (2547), cell (2291), cell part (2257), membrane part (2102), and organelle (1524). For KEGG pathway annotation (Figure 3), the top 10 pathways were signal transduction (1106), endocrine system (632), transport and catabolism (614), translation (559), cellular community (484), cell growth, and death (444), carbohydrate metabolism (436), folding, sorting and degradation (433), immune system (428), and lipid metabolism (406).

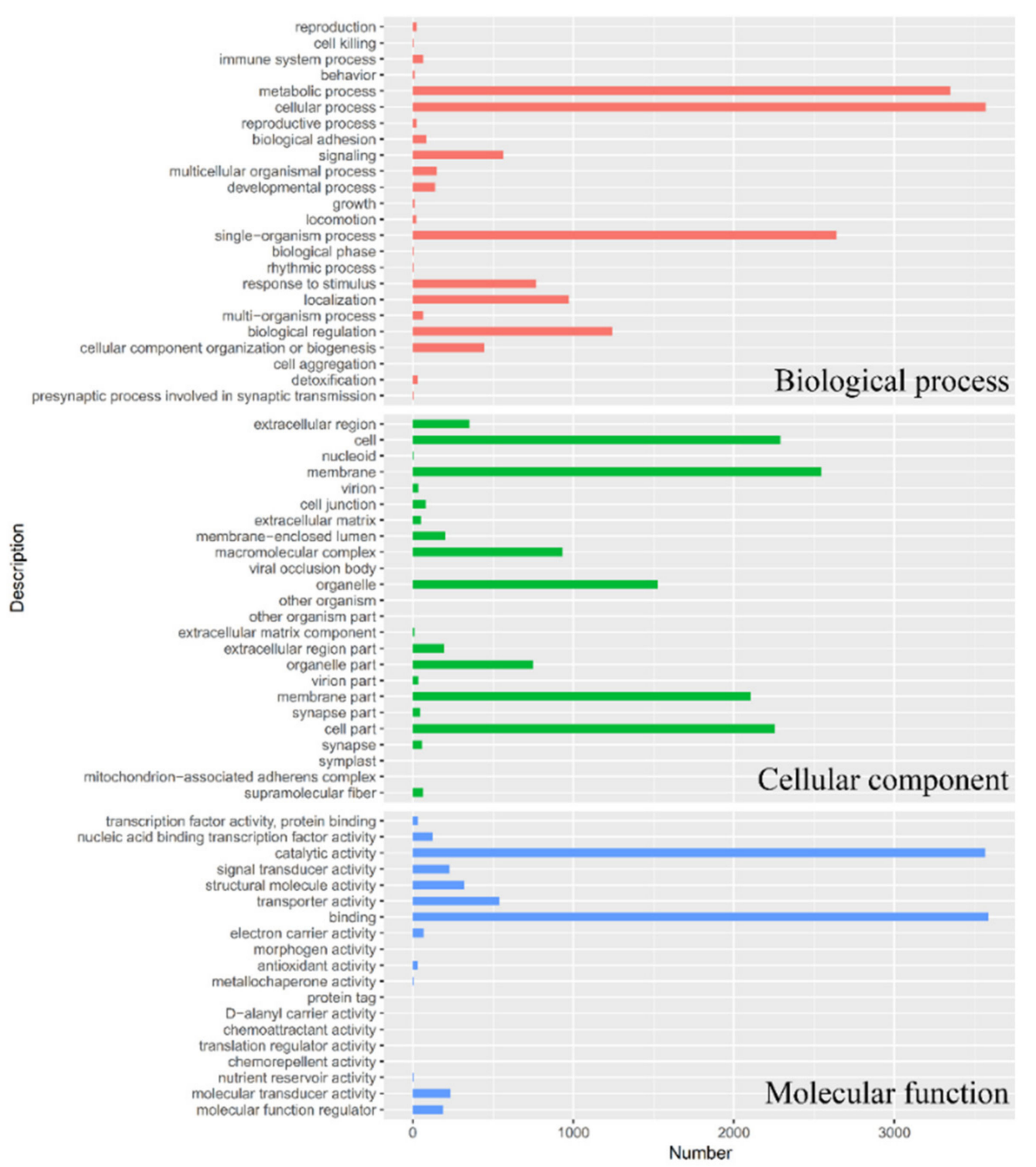

Figure 2. Gene Ontology (GO) classification analysis. Numbers of matched unigenes were plotted against each category for three main categories: biological process, cellular component, and molecular function. 


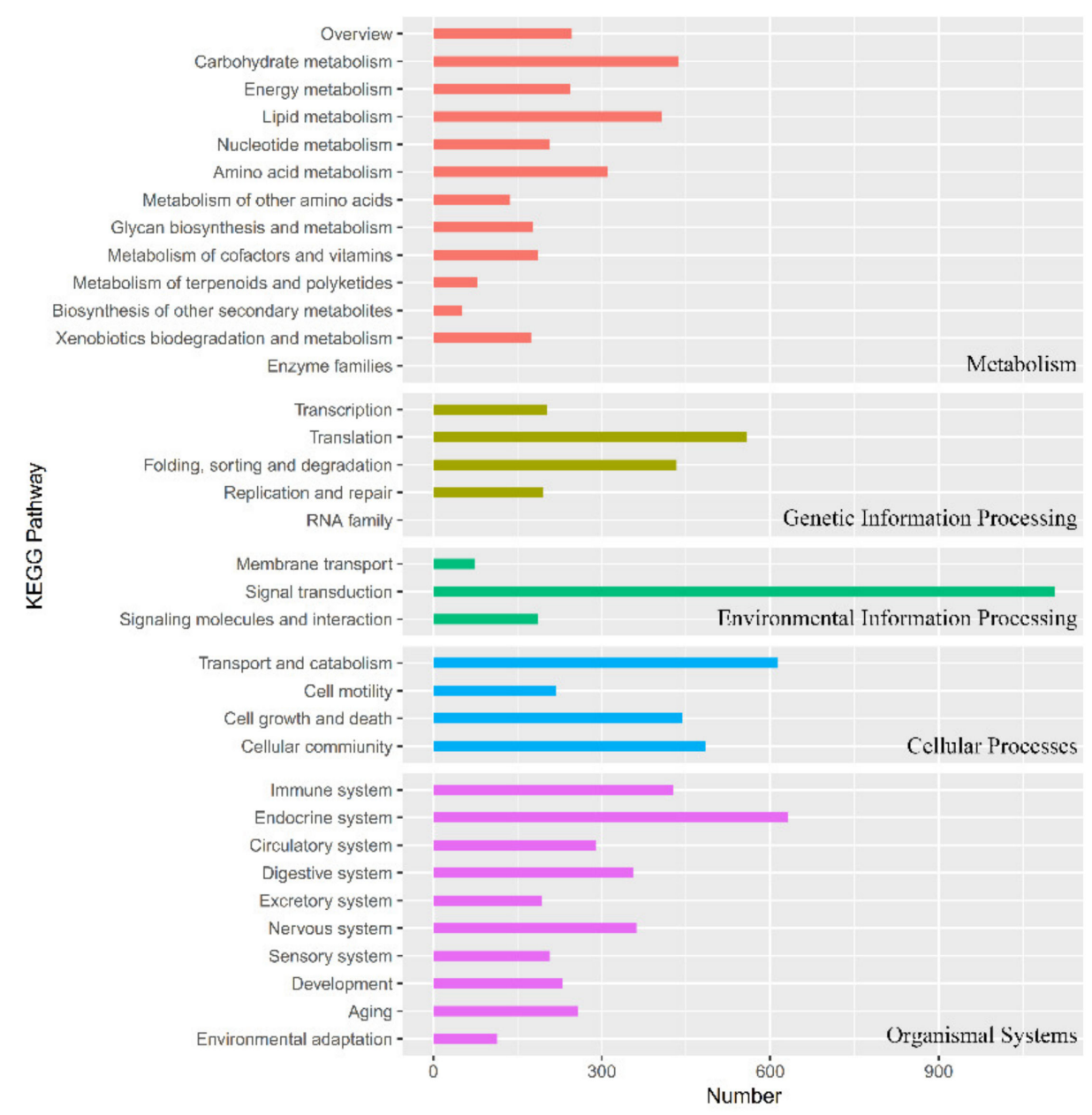

Figure 3. KEGG pathway annotation. KEGG Orthology (KO) and pathway annotations were completed by KEGG Automatic Annotation Server (KAAS) online automated annotation system. Numbers of matched unigenes were plotted against different KEGG pathways.

\subsection{Identification of CTLS}

Thirty-five unigenes that encode single-CRD or dual-CRD proteins were identified and named CTL-S1-CTL-S6 and IML-1-IML-29 (Figure 4A and Table 2). The coding sequences were amplified from cDNA and correctly sequenced (Figure 4B). To determine the phylogenetic relationships, 57 CTL-S and 90 IML were aligned to construct NJ trees, respectively [37]. All M. separata CTL-S clustered with their respective lepidopteran orthologs, except that CTL-S2 and CTL-S3 clustered together (Figure 5A). In contrast, IMLs showed lineage-specific expansions: IML-26/18/27/4/6/29/23/28/22/19 and IML-21/14/11/1/10/16/12/5/25/7/3/20/24/13/9 formed two clusters, indicating that they originated from independent expansions. IML-2 is a 1:1 ortholog of $H$. armigera (XP_021181291.1). IML-8 is a 1:1 ortholog of S. litura (XP_022819861.1). IML-17 is clustered with six $H$. armigera and seven $S$. litura orthologs (Figure 5B). These phylogenetic relationships are consistent with previous reports of B. mori and M. sexta CTLs $[7,8]$. 
A

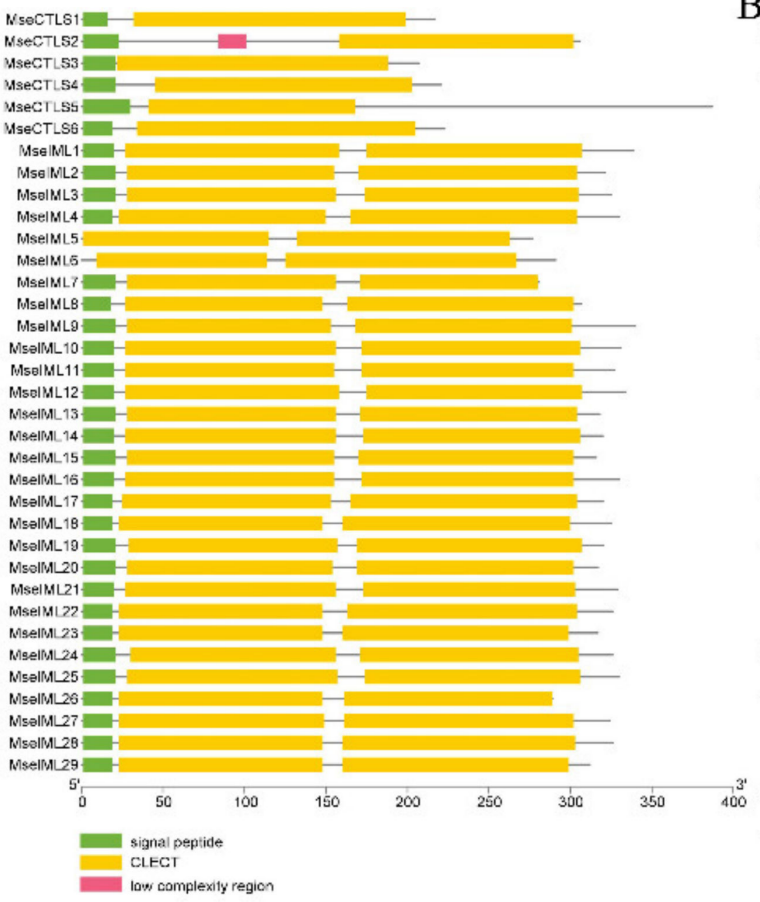

B
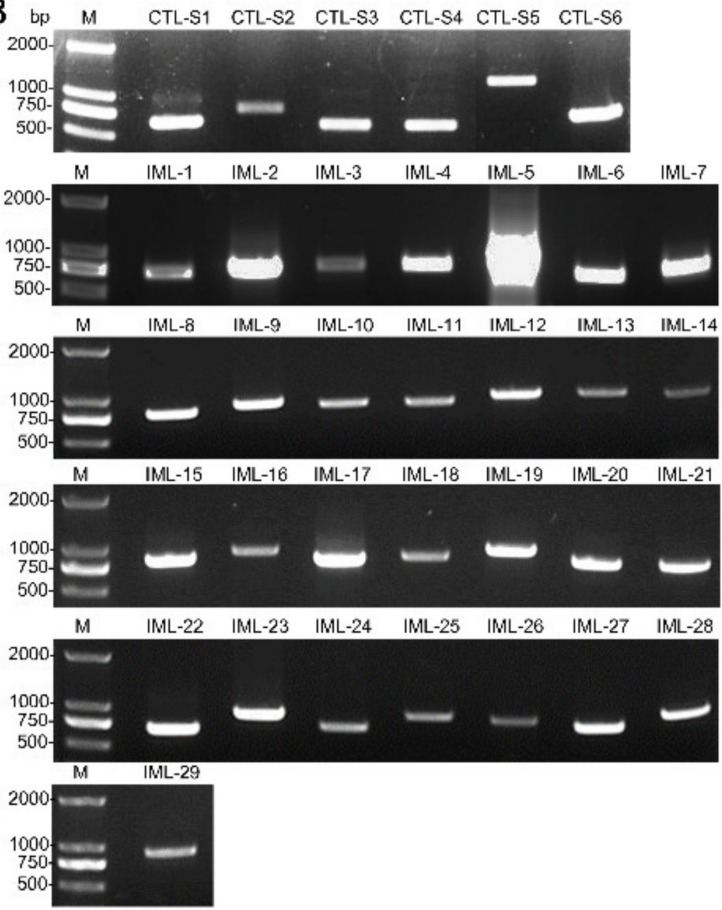

Figure 4. Domain architecture and PCR amplification of M. separata CTLs: (A) domain architecture of CTLs was analyzed by SMART (http:/ / smart.embl-heidelberg.de/) (accessed on 10 May 2021); and (B) CTL genes were amplified using primers in Table $\mathrm{S} 1$ and analyzed on $1 \%$ agarose gel.
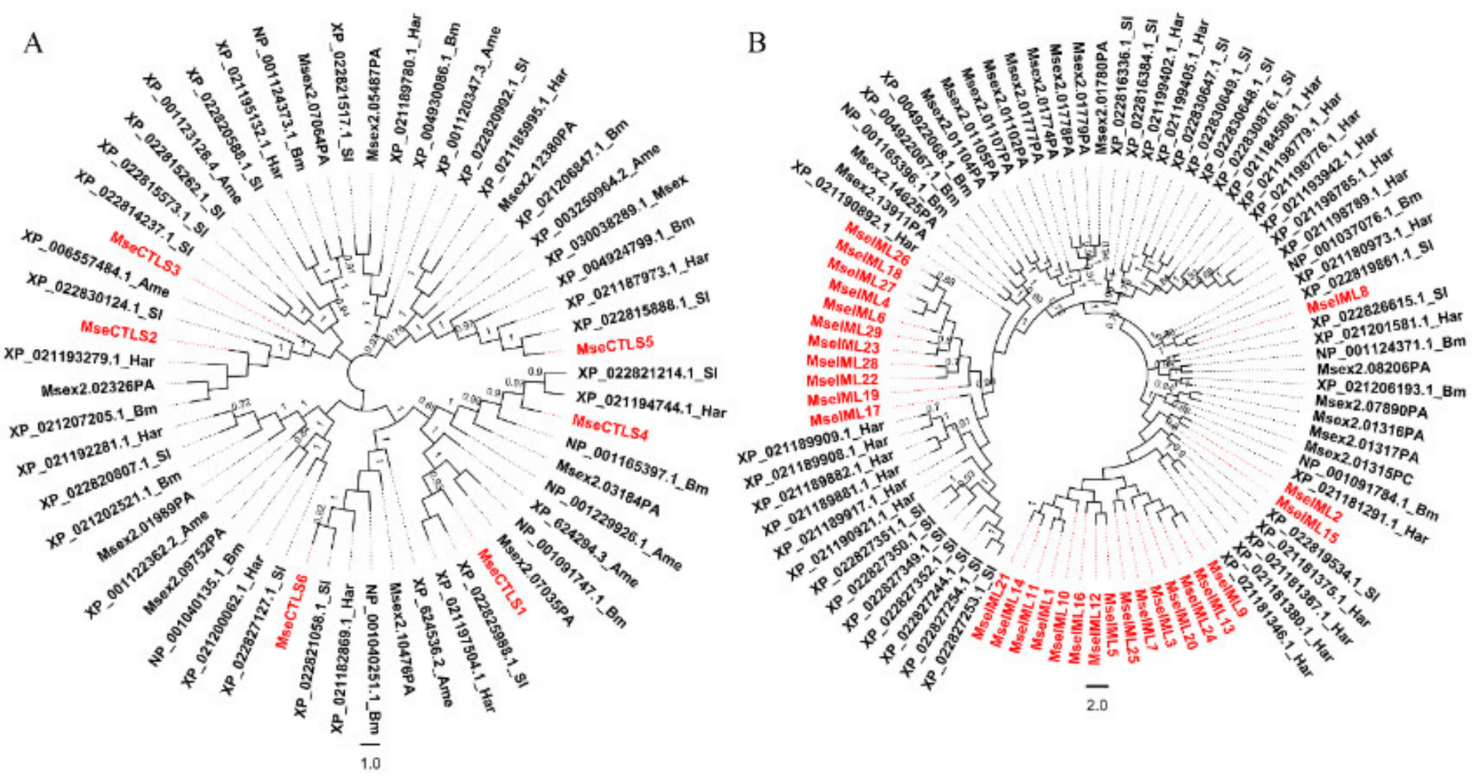

Figure 5. Phylogenetic trees of CTL-S and IML. Protein sequences of 57 CTL-S (A) and 90 IML (B) were aligned by MUSCLE (https:/ / www.ebi.ac.uk/Tools/msa/muscle/) (accessed on 10 May 2021). The neighbor-joining tree was constructed in MEGA $X$. The percentage $(>70 \%)$ of replicate trees in which the associated taxa clustered together in the bootstrap test (1000 replicates) were shown next to the branches as decimals. The evolutionary distances were computed using the p-distance method and are in the units of the number of amino acid differences per site. All ambiguous positions were removed for each sequence pair (pairwise deletion option). M. separata CTLs are shown in red. Msex: Manduca sexta; Bm: Bombyx mori; Ame: Apis mellifera; Sl: Spodoptera litura; Har: Helicoverpa armigera. 
Table 2. M. separata CTL genes identified from unigenes.

\begin{tabular}{|c|c|c|c|c|c|c|}
\hline \multirow{2}{*}{ Name } & \multirow{2}{*}{$\begin{array}{c}\text { Accession } \\
\text { Number }\end{array}$} & \multirow{2}{*}{ ORF (aa) } & \multicolumn{4}{|c|}{ BLASTX Best Hit } \\
\hline & & & Reference Organism & Accession Number & E Value & Identity (\%) \\
\hline CTL-S1 & MW658722 & 217 & Manduca sexta & XP_030027697.2 & $4 \times 10^{-147}$ & 99 \\
\hline CTL-S2 & MW658723 & 306 & Spodoptera litura & XP_022830124.1 & $2 \times 10^{-179}$ & 86 \\
\hline CTL-S3 & MW658724 & 207 & Helicoverpa armigera & XP_021189783.1 & $5 \times 10^{-143}$ & 100 \\
\hline CTL-S4 & MW658725 & 221 & Manduca sexta & XP_030021432.1 & $1 \times 10^{-149}$ & 95 \\
\hline CTL-S5 & MW658726 & 387 & Spodoptera litura & XP_022815888.1 & 0 & 89 \\
\hline CTL-S6 & MW658727 & 223 & Spodoptera litura & XP_022821058.1 & $3 \times 10^{-152}$ & 99 \\
\hline IML-1 & MW658728 & 339 & Spodoptera frugiperda & XP_035436562.1 & $6 \times 10^{-131}$ & 58 \\
\hline IML-2 & MW658729 & 321 & Spodoptera frugiperda & XP_035436562.1 & 0 & 77 \\
\hline IML-3 & MW658730 & 325 & Spodoptera exigua & KĀF9421847.1 & $6 \times 10^{-122}$ & 54 \\
\hline IML-4 & MW658731 & 330 & Mythimna separata & BBC20960.1 & $2 \times 10^{-165}$ & 71 \\
\hline IML-5 & MW658732 & 277 & Spodoptera exigua & AQX37248.1 & $6 \times 10^{-106}$ & 54 \\
\hline IML-6 & MW658733 & 291 & Mythimna separata & BBC20960.1 & $3 \times 10^{-118}$ & 62 \\
\hline IML-7 & MW658734 & 281 & Spodoptera frugiperda & XP_035436562.1 & $2 \times 10^{-104}$ & 53 \\
\hline IML-8 & MW658735 & 307 & Trichoplusia ni & XP_026724737.1 & $9 \times 10^{-150}$ & 65 \\
\hline IML-9 & MW658736 & 340 & Spodoptera frugiperda & XP_035436562.1 & $3 \times 10^{-110}$ & 51 \\
\hline IML-10 & MW658737 & 331 & Spodoptera exigua & AQX37248.1 & $2 \times 10^{-110}$ & 51 \\
\hline IML-11 & MW658738 & 327 & Spodoptera frugiperda & XP_035436562.1 & $1 \times 10^{-112}$ & 50 \\
\hline IML-12 & MW658739 & 334 & Spodoptera frugiperda & XP_035436562.1 & $8 \times 10^{-111}$ & 54 \\
\hline IML-13 & MW658740 & 318 & Spodoptera frugiperda & XP_035436562.1 & $4 \times 10^{-114}$ & 54 \\
\hline IML-14 & MW658741 & 320 & Spodoptera frugiperda & XP_035436562.1 & $2 \times 10^{-121}$ & 57 \\
\hline IML-15 & MW658742 & 316 & Spodoptera frugiperda & XP_035436562.1 & $3 \times 10^{-119}$ & 56 \\
\hline IML-16 & MW658743 & 330 & Spodoptera frugiperda & XP_035436562.1 & $3 \times 10^{-116}$ & 52 \\
\hline IML-17 & MW658744 & 320 & Mythimna separata & BAV01248.1 & 0 & 98 \\
\hline IML-18 & MW658745 & 325 & Mythimna separata & ВBC20960.1 & $1 \times 10^{-146}$ & 63 \\
\hline IML-19 & MW658746 & 320 & Mythimna separata & BAV01248.1 & $9 \times 10^{-127}$ & 59 \\
\hline IML-20 & MW658747 & 317 & Spodoptera frugiperda & XP_035436562.1 & $4 \times 10^{-121}$ & 56 \\
\hline IML-21 & MW658748 & 329 & Spodoptera frugiperda & XP_035436562.1 & $1 \times 10^{-120}$ & 54 \\
\hline IML-22 & MW658749 & 326 & Trichoplusia ni & XP_026732761.1 & $1 \times 10^{-112}$ & 53 \\
\hline IML-23 & MW658750 & 317 & Mythimna separata & BAV01248.1 & $2 \times 10^{-120}$ & 57 \\
\hline IML-24 & MW658751 & 326 & Spodoptera frugiperda & XP_035436562.1 & $8 \times 10^{-126}$ & 55 \\
\hline IML-25 & MW658752 & 330 & Spodoptera frugiperda & XP_035436562.1 & $1 \times 10^{-119}$ & 59 \\
\hline IML-26 & MW658753 & 289 & Mythimna separata & BBC20960.1 & $3 \times 10^{-12}$ & 62 \\
\hline IML-27 & MW658754 & 324 & Mythimna separata & ВВС20960.1 & 0 & 99 \\
\hline IML-28 & MW658755 & 326 & Mythimna separata & ВBC20960.1 & $2 \times 10^{-135}$ & 59 \\
\hline IML-29 & MW658756 & 312 & Trichoplusia ni & XP_026732761.1 & $1 \times 10^{-142}$ & 64 \\
\hline
\end{tabular}

\subsection{Sequence and Structure Analyses of CRDs}

M. separata CRDs were analyzed by I-TASSER for ligand type and interacting residues. Of 64 CRDs, 15 contained 'EPN' motifs, 12 contained 'QPD' motifs, and 37 had noncanonical motifs (Table 3). Sequence logos showed the conserved residues in each type of CRD. Four cysteines were completely conserved in all CRDs. Other conserved residues include: $\mathrm{E}^{26} / \mathrm{G}^{27} / \mathrm{L}^{30} / \mathrm{E}^{38} / \mathrm{P}^{53} / \mathrm{W}^{68} / \mathrm{G}^{75} / \mathrm{D}^{78} / \mathrm{G}^{83} / \mathrm{F}^{89} / \mathrm{T}^{91} / \mathrm{G}^{94} / \mathrm{Y}^{102} / \mathrm{W}^{105} / \mathrm{E}^{109} / \mathrm{P}^{110} / \mathrm{N}^{111} / \mathrm{E}^{121}$ $/ \mathrm{G}^{131} / \mathrm{D}^{138} / \mathrm{F}^{147} / \mathrm{I}^{148}$ in 'EPN'-type CRDs (Figure $6 \mathrm{~A}$ and Figure S1A); $\mathrm{E}^{44} / \mathrm{G}^{72} / \mathrm{G}^{86} /$ $\mathrm{F}^{88} / \mathrm{W}^{88} / \mathrm{T}^{90} / \mathrm{G}^{93} / \mathrm{W}^{111} / \mathrm{Q}^{121} / \mathrm{P}^{122} / \mathrm{D}^{123} / \mathrm{D}^{152} / \mathrm{N}^{152} / \mathrm{F}^{161} / \mathrm{I}^{162}$ in 'QPD'-type CRDs (Figure $6 \mathrm{~B}$ and Figure $\mathrm{S} 1 \mathrm{~B}$ ); $\mathrm{E}^{38} / \mathrm{L}^{42} / \mathrm{G}^{84} / \mathrm{H}^{92} / \mathrm{G}^{98} / \mathrm{T}^{107} / \mathrm{W}^{123} / \mathrm{F}^{184} / \mathrm{I}^{185}$ in 'noncanonical'type CRDs (Figure 6C and Figure S1C). In MBP-A, Glu ${ }^{185}$, Asn ${ }^{187}, \mathrm{Glu}^{193}, \mathrm{Asn}^{205}$, and $\mathrm{Asp}^{206}$ can form coordination and hydrogen bonds with $\mathrm{Ca}^{2+}$ and 3-OH/4-OH, respectively (Figure 6D). The sequence alignment shows that in 'EPN'-type CRDs, Glu ${ }^{193}$ is completely conserved, $\mathrm{Asn}^{205}$ is conserved in 10 out of 15 CRDs, and Asp ${ }^{206}$ is conserved in 14 out of 15 CRDs. In the MBP-A mutant (QPDWG), Gln ${ }^{185}-$ Asp $^{187}-\mathrm{Glu}^{198}-\mathrm{Asn}^{210}-\mathrm{Asp}^{211}$ forms a similar coordination and hydrogen bond network. However, the reversed hydrogen donor/acceptor and the adjacent $\operatorname{Tr}^{189}$ switched the preferred ligand to galactose (Figure 6E). The sequence comparison showed that $\operatorname{Tr}^{189}$ and $\mathrm{Asn}^{210}$ were not conserved in insect CRDs. Glu ${ }^{198}$ was conserved in 5 out of 12 CRDs. Asp ${ }^{211}$ was conserved in 8 out of 12 CRDs. In the predicted structures of IML-10-CRD1 ('EPN', Figure 6F), IML-14-CRD1 ('QPD', Figure 6G), and IML-17-CRD1 ('EPD', Figure 6H), sugar ligands were accom- 
modated in proper orientations and positions through hydrogen bonds formed between hydroxyl groups and amino acids.

Table 3. Sequence features of CRDs.

\begin{tabular}{|c|c|c|c|c|c|c|}
\hline CRD & Motif & PDB Hit & $\mathrm{Ca}^{2+}$ Coordinators ${ }^{2}$ & Ligand $^{1}$ & Ligand Binding Residues $^{2}$ & Disulfide Bridges \\
\hline CTL-S1 & QPD & $1 \mathrm{dv} 8 \mathrm{~A}$ & $38,40,44,158[0.14]$ & GQ2 & $59,115,117,118,132,145,146,147,153[0.48]$ & $70-198 ; 171-190$ \\
\hline CTL-S2 & EPN & $4 \mathrm{kzvA}$ & $62,66,90,99,100[0.39]$ & MAN & $87,89,99,115,116,118[0.87]$ & $197-301 ; 275-293$ \\
\hline CTL-S3 & VPQ & $1 \mathrm{htnA}$ & $36,38,42,160[0.13]$ & 1SL5A & $\begin{array}{c}104,105,106,107,108,109,110,111,116,117 \\
119,120,121,122,123,124,125,126,148,153[0.24]\end{array}$ & $55-187 ; 164-179$ \\
\hline CTL-S4 & QPD & $4 \mathrm{kzvA}$ & $35,37,41,154[0.13]$ & 4RS & $56,112,114,124,130,141,142,147[0.51]$ & $75-202 ; 175-194$ \\
\hline CTL-S5 & - & 1wmyA & $31,33,37,121[0.12]$ & GQ2 & $53,85,87,88,98,108,109,110,116[0.35]$ & $69-167 ; 143-159$ \\
\hline CTL-S6 & QPD & $4 \mathrm{kzvA}$ & $38,40,44,163[0.10]$ & BM3 & $120,122,124,133,150,151,152[0.50]$ & $71-204 ; 177-196$ \\
\hline IML-1-CRD1 & QPD & $4 \mathrm{kzvA}$ & $56,60,85,90,91[0.44]$ & NGA & $49,82,84,86,90,94,96,105,106[0.57]$ & $57-157 ; 132-149$ \\
\hline IML-1-CRD2 & QPD & $4 y l i A$ & $31,33,37,126[0.22]$ & 291 & $93,95,101,107,113,114,115[0.32]$ & $203-306 ; 284-298$ \\
\hline IML-2-CRD1 & EPN & 4yliA & $55,59,62,84,89,90[0.27]$ & MAN & $81,83,89,101,102,104[0.73]$ & $58-154 ; 132-146$ \\
\hline IML-2-CRD2 & QPD & 1wmyA & $31,33,37,126[0.13]$ & 4RS & $61,94,96,101,107,113,114,119[0.66]$ & $200-303 ; 281-295$ \\
\hline IML-3-CRD1 & QPD & 4yliA & $56,60,85,90,91[0.38]$ & GQ2 & $49,82,84,86,94,102,103,104,110[0.58]$ & $58-155 ; 133-147$ \\
\hline IML-3-CRD2 & QPE & 4yliA & $31,33,37,125[0.16]$ & 4RS & $61,93,95,100,106,112,113,118[0.51]$ & $202-304 ; 282-296$ \\
\hline IML-4-CRD1 & EPD & 4yliA & $24,26,30,114[0.03]$ & MAN & $81,83,89,101,102,104[0.81]$ & $51-149 ; 127-141$ \\
\hline IML-4-CRD2 & EPN & 4yliA & $31,33,37,131[0.11]$ & 4RS & $64,98,100,106,112,118,119,124[0.64]$ & $195-303 ; 281-295$ \\
\hline IML-5-CRD1 & QPD & 4yliA & $56,60,63,85,90,91[0.10]$ & TRE & $49,82,84,86,90,96,102,103,108[0.67]$ & $17-114 ; 92-106$ \\
\hline IML-5-CRD2 & KPA & 4yliA & $29,31,35,123[0.23]$ & BM3 & $92,94,96,98,110,111,112[0.21]$ & $160-262 ; 240-254$ \\
\hline IML-6-CRD1 & EPD & 4yliA & $37,41,44,66,71,72[0.15]$ & NAG & $63,65,71,83,84,89[0.79]$ & $19-114 ; 91-105$ \\
\hline IML-6-CRD2 & $\mathrm{EPN}$ & 4yliA & $31,33,37,131[0.11]$ & MAN & $61,98,100,106,118,119,121[0.67]$ & $158-266 ; 244-258$ \\
\hline IML-7-CRD1 & QPS & 4yliA & $61,65,90,95,96[0.38]$ & MAN & $87,89,95,107,108,110[0.65]$ & $58-155 ; 133-147$ \\
\hline IML-7-CRD2 & LPQ & 4yliA & $66,70,72,95,98,99[0.15]$ & MAN & $92,94,96,98[0.39]$ & 201-?; 281-? \\
\hline IML-8-CRD1 & NTD & $1 \mathrm{htn} A$ & $40,42,46,122[0.16]$ & NGA & $63,89,90,91,92,109,110,111[0.23]$ & $57-147 ; 125-139$ \\
\hline IML-8-CRD2 & $\mathrm{EPN}$ & 1 jznA & $27,29,33,127[0.10]$ & MAN & $54,93,95,101,114,115,117[0.72]$ & $193-301 ; 278-293$ \\
\hline IML-9-CRD1 & HPD & 4 yliA & $31,33,37,117[0.16]$ & 4RS & $54,87,89,92,98,104,105,110[0.45]$ & $58-152 ; 130-144$ \\
\hline IML-9-CRD2 & QAD & 4yliA & $31,33,37,125[0.22]$ & GQ2 & $61,92,94,95,104,112,113,114,120[0.34]$ & $198-300 ; 278-292$ \\
\hline IML-10-CRD1 & EPN & $4 y l i A$ & $56,60,85,90,91[0.22]$ & MÂN & $82,84,90,103,104,106[0.71]$ & $57-155 ; 132-147$ \\
\hline IML-10-CRD2 & QPD & 4yliA & $29,31,35,125[0.20]$ & NGA & $59,92,94,96,100,104,106,112,113[0.20]$ & $201-305 ; 283-297$ \\
\hline IML-11-CRD1 & TPD & 4yliA & $56,60,85,90,91[0.24]$ & MAN & $82,84,90,102,103,105[0.62]$ & $57-154 ; 132-146$ \\
\hline IML-11-CRD2 & KPD & 4yliA & $31,33,37,124[0.14]$ & GAL & $94,96,99,111,112[0.21]$ & $200-301 ; 279-293$ \\
\hline IML-12-CRD1 & TPD & $4 \mathrm{kzvA}$ & $56,60,85,90,91[0.27]$ & NGA & $49,82,84,86,90,94,96,105,106[0.55]$ & $57-157 ; 132-149$ \\
\hline IML-12-CRD2 & EPD & 4yliA & $31,33,37,126[0.19]$ & GQ2 & $61,93,95,96,105,113,114,115,121[0.52]$ & $203-306 ; 284-298$ \\
\hline IML-13-CRD1 & $\mathrm{EPN}$ & 4yliA & $57,61,86,90,91[0.23]$ & MAN & $83,85,90,102,103,105[0.69]$ & $59-155 ; 133-147$ \\
\hline IML-13-CRD2 & QPD & 4yliA & $31,33,37,125[0.21]$ & 4RS & $61,93,95,100,106,112,113,118[0.47]$ & $201-303 ; 281-295$ \\
\hline IML-14-CRD1 & QPD & 4yliA & $56,60,63,86,91,92[0.35]$ & MAN & $83,85,91,103,104,106[0.67]$ & $57-155 ; 133-147$ \\
\hline IML-14-CRD2 & QPD & 4yliA & $31,33,37,127[0.32]$ & GQ2 & $61,94,96,97,106,114,115,116,122[0.09]$ & $201-305 ; 283-297$ \\
\hline IML-15-CRD1 & $\mathrm{EPN}$ & 4yliA & $56,60,63,85,89,90[0.29]$ & MAN & $82,84,89,101,102,104[0.70]$ & $58-154 ; 132-146$ \\
\hline IML-15-CRD2 & SRL & 4yliA & $31,33,37,124[0.24]$ & GQ2 & $61,91,93,94,103,111,112,113,119[0.30]$ & $200-301 ; 279-293$ \\
\hline IML-16-CRD1 & EPN & 4 yliA & $56,60,85,90,91[0.23]$ & MĀN & $82,84,90,102,103,105[0.70]$ & $57-154 ; 132-146$ \\
\hline IML-16-CRD2 & QPV & 4yliA & $31,33,37,124[0.13]$ & MMA & $94,96,98,99,111,112,113[0.18]$ & $200-301 ; 279-293$ \\
\hline IML-17-CRD1 & EPD & 4yliA & $18,20,24,109[0.05]$ & MAN & $76,78,84,96,97,99[0.79]$ & $53-152 ; 130-144$ \\
\hline IML-17-CRD2 & $\mathrm{EPN}$ & 4yliA & $31,33,37,128[0.11]$ & TRE & $61,95,97,99,103,109,115,116,121[0.63]$ & $198-303 ; 281-295$ \\
\hline IML-18-CRD1 & EPD & 4yliA & $18,20,24,106[0.04]$ & MAN & $73,75,81,93,94,96[0.78]$ & $51-147 ; 125-139$ \\
\hline IML-18-CRD2 & EPT & 4yliA & - & 4RS & $63,97,99,104,110,116,117,122[0.68]$ & $193-299,277-291$ \\
\hline IML-19-CRD1 & EPD & 4yliA & $26,28,32,117[0.04]$ & TRE & $51,84,86,88,92,98,104,105,110[0.79]$ & $57-156 ; 134-148$ \\
\hline IML-19-CRD2 & NPD & $1 \mathrm{~b} 08 \mathrm{~A}$ & $31,33,37,127[0.18]$ & GQ2 & $61,95,97,98,106,114,115,116,122[0.56]$ & $202-306 ; 284-298$ \\
\hline IML-20-CRD1 & QPN & 4yliA & $26,28,32,113[0.07]$ & MAN & $82,84,89,100,101,103[0.66]$ & $58-153 ; 132-145$ \\
\hline IML-20-CRD2 & QPI & 4yliA & $31,33,37,126[0.15]$ & NGA & $94,96,98,101,113,114,115[0.41]$ & $198-301 ; 279-293$ \\
\hline IML-21-CRD1 & $\widehat{I P D}$ & 4yliA & $56,60,86,91,92[0.23]$ & TRE & $49,83,85,87,91,97,103,104,109[0.62]$ & $57-155 ; 133-147$ \\
\hline IML-21-CRD2 & QPV & 1wmyA & $31,33,37,124[0.21]$ & NAG & $91,93,100,111,112,113[0.35]$ & $201-302 ; 280-294$ \\
\hline IML-22-CRD1 & EPD & 4yliA & $26,28,32,114[0.04]$ & TRE & $48,81,83,85,89,95,101,102,107[0.81]$ & $51-147 ; 125-139$ \\
\hline IML-22-CRD2 & KPT & 4yliA & $31,33,37,133[0.17]$ & GQ2 & $67,101,103,104,112,120,121,122,128[0.54]$ & $193-303 ; 281-295$ \\
\hline IML-23-CRD1 & EPD & 4yliA & $26,28,32,114[0.04]$ & MAN & $81,83,89,101,102,104[0.81]$ & $51-147 ; 125-139$ \\
\hline IML-23-CRD2 & $\mathrm{DPN}$ & 4yliA & $31,33,37,129[0.10]$ & 4RS & $61,95,97,104,110,116,117,122[0.64]$ & $192-298 ; 276-290$ \\
\hline IML-24-CRD1 & EPN & 4yliA & $56,60,85,90,91[0.36]$ & MAN & $82,84,90,102,103,105[0.68]$ & $58-155 ; 133-147$ \\
\hline IML-24-CRD2 & VPT & 4yliA & $31,33,37,126[0.13]$ & GQ2 & $61,94,96,97,105,113,114,115,121[0.27]$ & $201-304 ; 282-296$ \\
\hline IML-25-CRD1 & EPN & 4yliA & $57,61,64,86,91,92[0.16]$ & MAN & $83,85,91,103,104,106[0.69]$ & $58-156 ; 134-148$ \\
\hline IML-25-CRD2 & QPG & 4yliA & $29,31,35,124[0.19]$ & GQ2 & $59,92,94,95,103,111,112,113,119[0.31]$ & $202-305 ; 283-297$ \\
\hline IML-26-CRD1 & EPD & $4 \mathrm{kzvA}$ & $34,36,40,122[0.04]$ & NGA & $56,89,91,93,97,101,103,109,110[0.78]$ & $51-147 ; 125-139$ \\
\hline IML-26-CRD2 & EPT & 3zhgA & - & MMA & $97,99,101,104[0.23]$ & 194-?; 278-? \\
\hline IML-27-CRD1 & EPD & 4yliA & $24,26,30,113[0.04]$ & MAN & $80,82,88,100,101,103[0.80]$ & $51-148 ; 126-140$ \\
\hline IML-27-CRD2 & EPN & 4yliA & $31,33,37,130[0.14]$ & FUC & $98,100,102,105,117,118,119[0.32]$ & $194-301 ; 279-293$ \\
\hline IML-28-CRD1 & EPD & 4yliA & $24,26,30,112[0.04]$ & MAN & $79,81,87,99,100,102[0.81]$ & $51-147 ; 125-139$ \\
\hline IML-28-CRD2 & EPN & 4yliA & $31,33,37,133[0.14]$ & 4RS & $67,101,103,108,114,120,121,126[0.59]$ & $192-302 ; 280-294$ \\
\hline IML-29-CRD1 & EPD & 4yliA & $26,28,32,114[0.04]$ & NGA & $48,81,83,85,89,93,95,101,102[0.80]$ & $51-147 ; 125-139$ \\
\hline IML-29-CRD2 & EPN & 4yliA & $68,72,75,98,103,104[0.03]$ & TRE & $61,95,97,99,103,109,115,116,121[0.65]$ & $193-298 ; 276-290$ \\
\hline
\end{tabular}

${ }^{1}$ GQ2: 6-o-alpha-d-glucopyranosyl-4-o-sulfo-alpha-d-glucopyranose; MAN: alpha-d-mannose; 1SL5A: alpha-l-fucopyranose-(1-3)-[beta-dgalactopyranose-(1-4)]2-acetamido-2-deoxy-beta-d-glucopyranose-(1-3)-beta-d-galactopyranose; 4RS: 6-butanoyl-trehalose; BM3: n-acetylalpha-d-mannosamine; NGA: n-acetyl-d-galactosamine; 291: prop-2-en-1-yl 7-o-carbamoyl-1-glycero-alpha-d-manno-heptopyranoside; TRE: trehalose; NAG: aldehydo-n-acetyl-d-glucosamine; GAL: beta-d-galactose; MMA: methyl alpha-d-mannopyranoside; FUC: alpha-1fucopyranose. ${ }^{2} \mathrm{C}$-scores of predicted $\mathrm{Ca}^{2+}$ and sugar ligands are shown in []. C-score is the confidence score of the prediction [0-1], where a higher score indicates a more reliable prediction. 


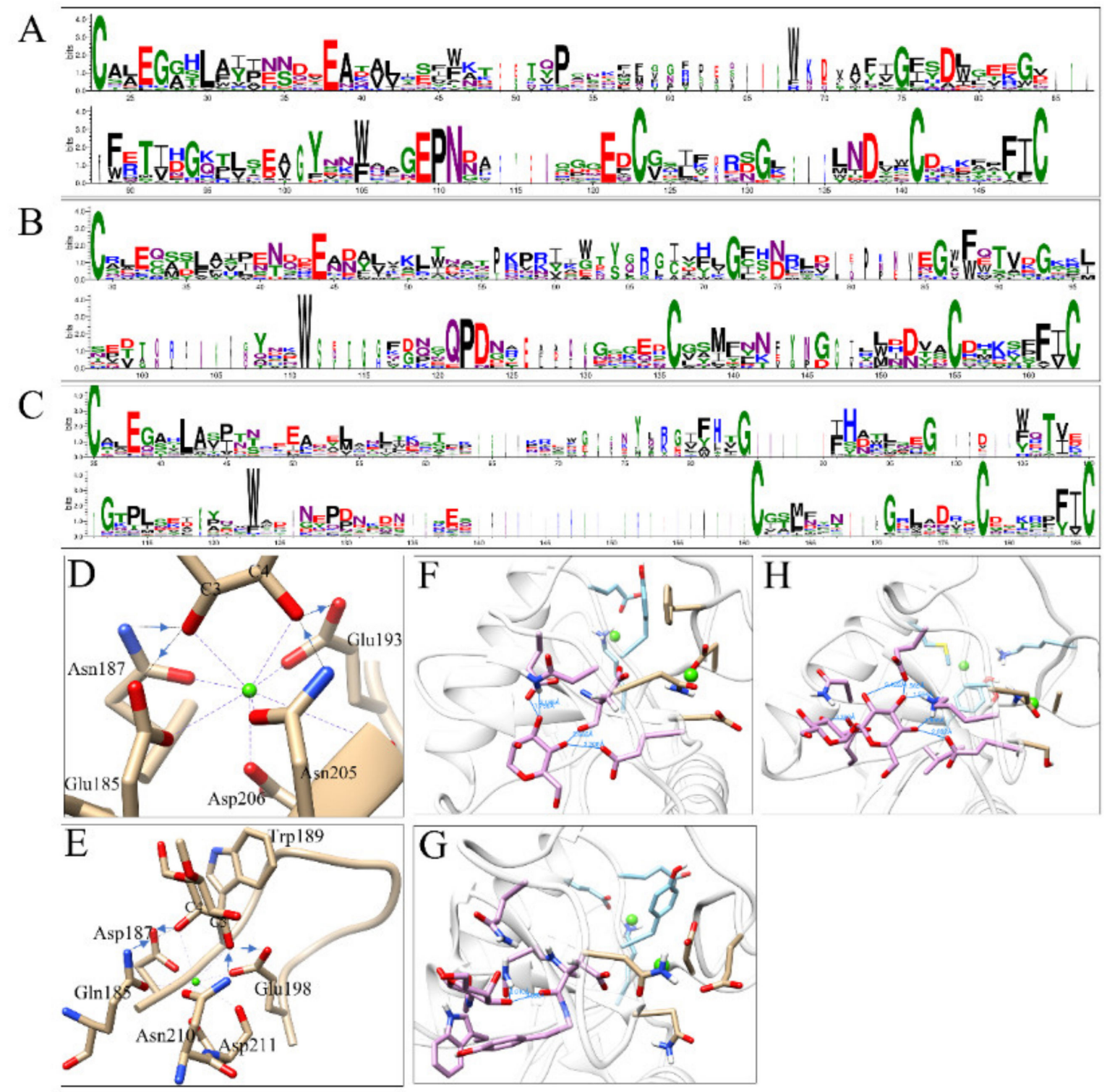

Figure 6. Sequence logos and structures of CRDs. Sequence logos of the 'EPN-type' (A); 'QPD-type' (B); and 'noncanonicaltype' (C) CRDs were generated using WebLogo. Amino acids were colored according to chemical properties: polar, green; neutral, purple; basic, blue; acidic, red; hydrophobic, black. The sugar-binding sites of rat MBP-A (PDB: 2MSB) (D) and the MBP-A (QPDWG) mutant (PDB: 1AFA) (E). The coordination bonds and hydrogen bonds were shown as purple and black dashed lines, respectively. $\mathrm{Ca}^{2+}$ ions were shown as green dots. Blue arrows indicate the direction of hydrogen bonds from the hydrogen donors to acceptors. The predicted structures of 'EPN' IML-10-CRD1 (F), 'QPD' IML-14-CRD1 (G), and 'EPD' IML-17-CRD1 (H). Hydrogen bonds were shown as blue lines with distance $(\AA)$.

\subsection{Spatial and Temporal Expression Profiles}

To explore the possible functions of CTLs, the expression profile in different developmental stages was analyzed by RT-qPCR. The hierarchical clustering analysis shows distinct expression patterns: CTL-S1, CTL-S2, CTL-S4, CTL-S5, and CTL-S6 mainly express in eggs and early stage larvae; IML-4, IML-5, and IML-18 express in adults; IML-3, IML-8, IML-21, CTL-S3, IML-14, IML-1, IML-28, IML-11, and IML-27 mainly express in pupa; IML-6, IML-16, IML-7, IML-25, IML-17, IML-20, and IML-24 express in late-stage larvae; IML-22, IML-29, IML-10, IML-12, IML-2, IML-15, IML-26, IML-19, IML-23, IML-9, and IML-13 express in early and mid-stage larvae (Figure 7A). Larval tissues can produce lots of immune factors to resist the invasion of pathogens. Thus, the expression profile in 
larval hemocytes, fat body, midgut, Malpighian tube, and epidermis was analyzed. The clustering analysis shows that hemocytes, fat body, and epidermis are the major tissues expressing CTL genes. Notably, IML-14 and IML-29 are mostly expressed in the midgut (Figure 7B).
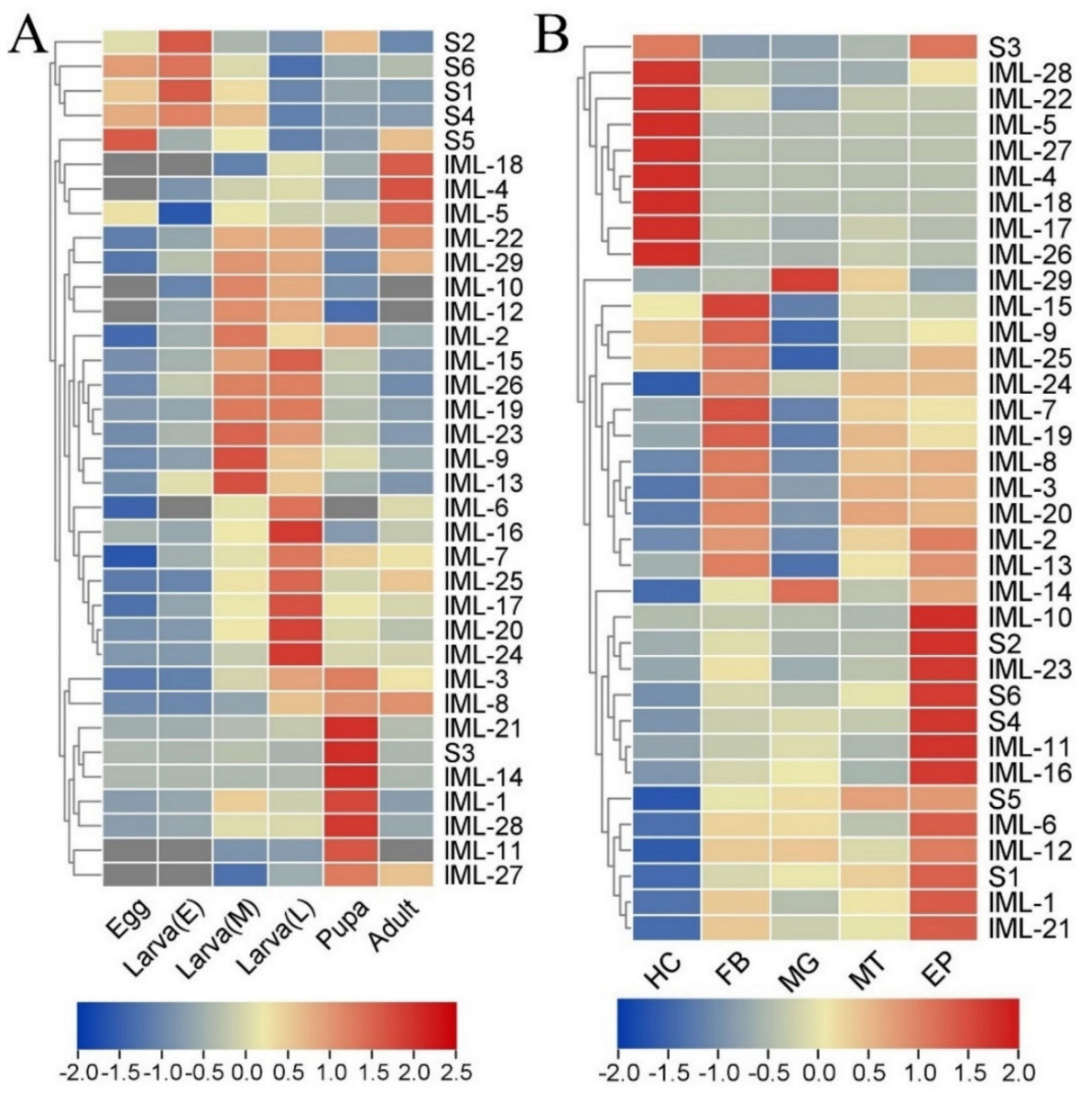

Figure 7. Temporal and spatial expression patterns of $M$. separata CTLs: (A) the expression profile of 35 CTLs in eggs, early stage larvae (E), mid-stage larvae (M), late-stage larvae (L), pupa, and adults; (B) the expression profile of 35 CTLs in larval tissues. HC, hemocytes; FB, fat body; MG, midgut; MT, Malpighian tube; EP, epidermis. Values $(\log 2)$ representing the relative expression levels were normalized for each gene (row) and mapped to the color scale on the bottom. Dendrograms were generated by the hierarchical clustering analysis of rows. Genes displaying similar expression patterns were recursively merged into clusters.

\subsection{Expression of CTLs Responding to Bacterial Cells and Fungal Spores}

C-type lectins, especially immulectins, are important for immune responses against bacteria and fungi $[17,38]$. The expression of CTLs in larval hemocytes, fat body, and midgut responding to E. coli, S. aureus, and B. bassiana were analyzed $6 \mathrm{~h}$ and $24 \mathrm{~h}$ postchallenge. At $6 \mathrm{hpi}$, in hemocytes, IML-20, IML-24, IML-21, and IML-16 were induced by $E$. coli; IML-21, IML-1, IML-29, and IML-26 were induced by S. aureus (Figure 8A); CTL-S1, CTL-S6, IML-1, and IML-11 were induced by B. bassiana (Figure 8B). In the fat body, IML-17, IML-10, IML-21, IML-6, IML-4, and IML-12 were induced by E. coli; IML-4, IML-8, IML-9, and $I M L-12$ were induced by S. aureus (Figure $8 C$ ); IML-8 and IML-19 were induced by $B$. bassiana (Figure 8D). In the midgut, IML-3, IML-4, IML-25, IML-13, IML-17, IML-14, IML-22, $I M L-10$, and IML-16 were induced by E. coli; IML-20 and IML-8 were induced by S. aureus (Figure 8E); IML-2, IML-16, and IML-23 were induced by B. bassiana (Figure $8 \mathrm{~F}$ ). At 24 hpi, in hemocytes, IML-4 and IML-16 were induced by E. coli; IML-18, IML-19, IML-13, IML-15, $I M L-2, I M L-8, I M L-3, I M L-24$, and IML-4 were induced by S. aureus (Figure 9A); IML-24 and $I M L-20$ were induced by B. bassiana (Figure 9B). In the fat body, IML-4 and IML-17 were induced by E. coli; IML-4, IML-17, IML-21, IML-28, and IML-18 were induced by S. aureus 
(Figure 9C); IML-23 and $I M L-24$ were induced by B. bassiana (Figure 9D). In the midgut, IML-4, IML-19, IML-11, CTL-S1, and CTL-S3 were induced by E. coli; IML-4 and CTL-S1 were induced by S. aureus (Figure 9E); IML-16, IML-12, IML-15, IML-23, and CTL-S6 were induced by B. bassiana (Figure 9F).
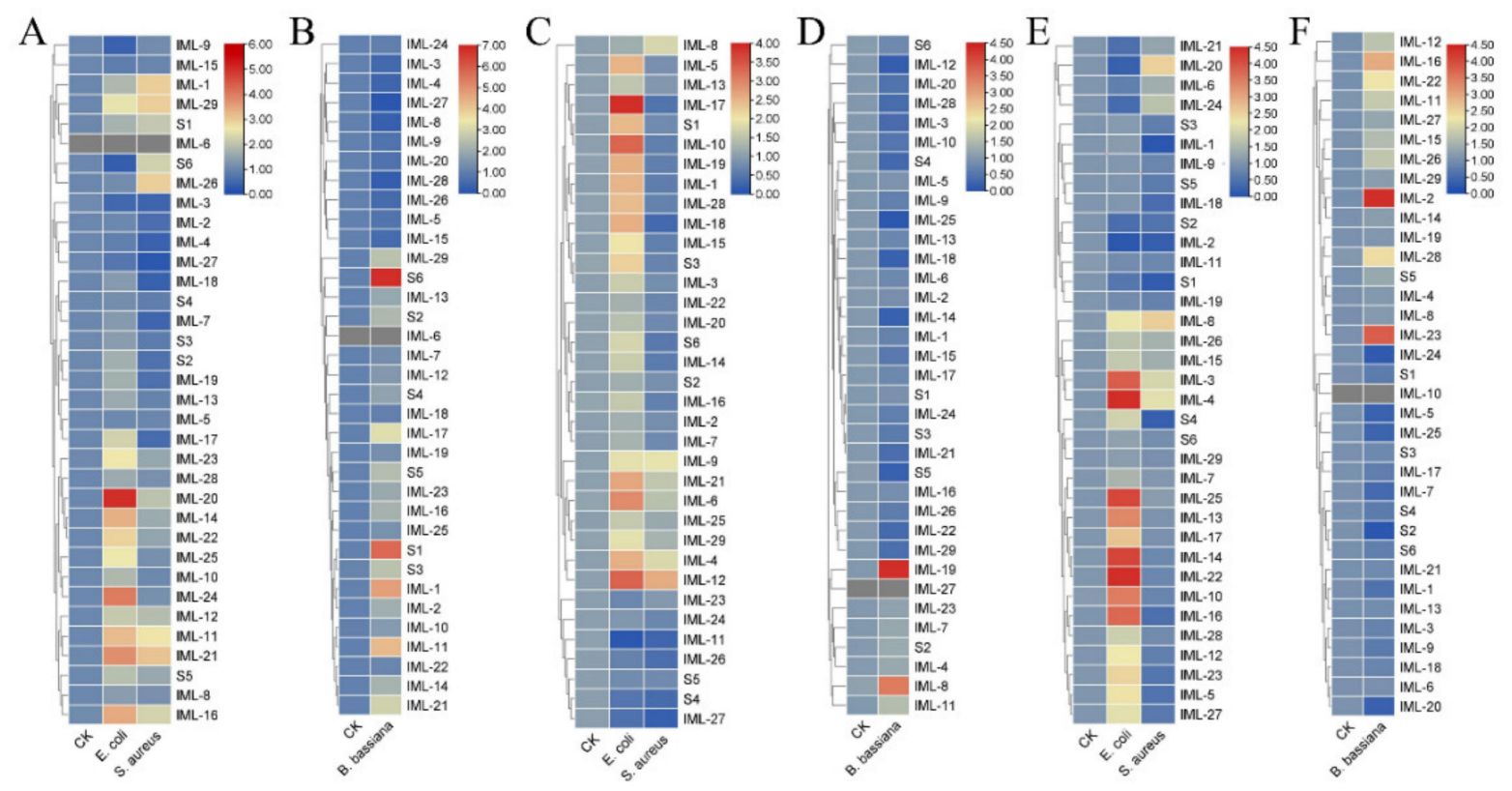

Figure 8. Expression of CTLs responding to E. coli, S. aureus, and B. bassiana conidia after 6 h. Formaldehyde-killed E. coli, S. aureus, and B. bassiana conidia were injected into fifth instar larvae. The expression of CTLs in hemocytes $(\mathbf{A}, \mathbf{B})$, fat body (C,D), and midgut (E,F) were analyzed by RT-qPCR. Values $(\log 2)$ representing the relative expression levels were directly mapped to the color scale without normalization. Dendrograms were generated by the hierarchical clustering analysis of rows.
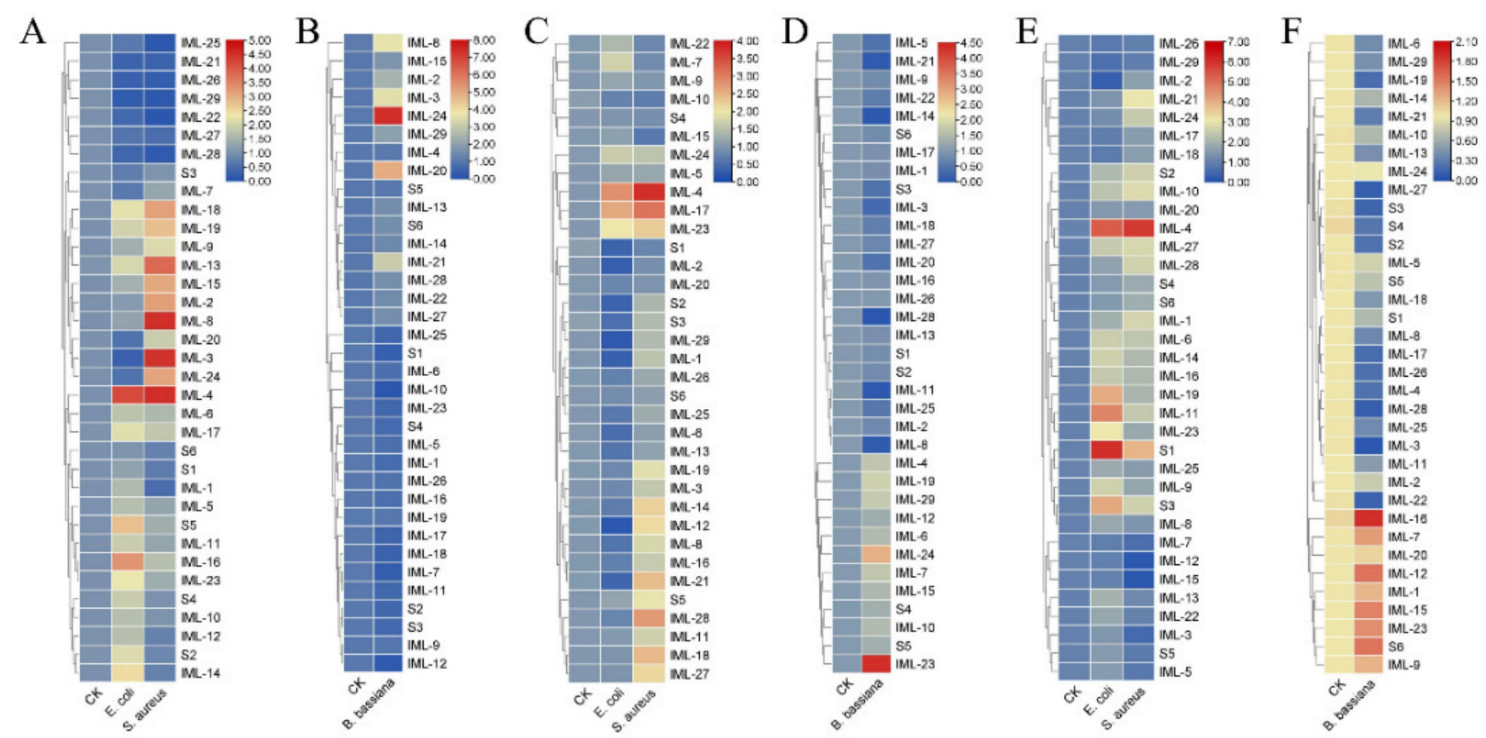

Figure 9. Expression of CTLs responding to E. coli, S. aureus, and B. bassiana conidia after 24 h. Formaldehyde-killed E. coli, S. aureus, and B. bassiana conidia were injected into fifth instar larvae. The expression of CTLs in hemocytes (A,B), fat body $(\mathbf{C}, \mathbf{D})$, and midgut $(\mathbf{E}, \mathbf{F})$ were analyzed by RT-qPCR. Values $(\log 2)$ representing the relative expression levels were directly mapped to the color scale without normalization. Dendrograms were generated by the hierarchical clustering analysis of rows. 


\section{Discussion}

With the advancement of the next-generation sequencing (NGS) technique, RNA-seq has become an indispensable tool for studying the transcriptome of non-model organisms, including some agricultural pests [39]. In this study, the transcriptome generated 44,966,148 clean reads and 45,888 unigenes with a mean length of $910 \mathrm{bp}$, which was comparable to the previous M. separata transcriptomes [40-47]. Most homologs of $M$. separata transcripts were found in lepidopteran species, especially in A. transitella and B. mori. By Gene Ontology (GO) classification and KEGG pathway annotation, unigenes were classified into a variety of biological processes, cellular components, molecular functions, and pathways.

Animal C-type lectin-like domain-containing proteins can be classified into 16 groups based on domain architecture and phylogenetic relationships [48]. In insects, CTLs can be classified based on domain architecture into CTL-S, IML, and CTL-X. CTL-S exist in several insect orders: Lepidoptera, Coleoptera, Hymenoptera, Diptera, and Hemiptera. The numbers of CTL-S vary in different species: Bombyx mori (12), Manduca sexta (8), Tribolium castaneum (10), Drosophila melanogaster (30), Anopheles gambiae (21), Aedes aegypti (37), Acyrthosiphon pisum (2), and Plutella xylostella (5). Immulectins were almost entirely found in Lepidoptera: Bombyx mori (6) and Manduca sexta (19) [5]. Here, we identified 6 CTL-S and 29 immulectins from the unigenes of $M$. separata larvae. The phylogenetic analysis showed that CTL-S genes were duplicated in the common ancestor before speciation (Figure 5A), while most IML genes were duplicated after speciation (Figure 5B). Similar phylogenetic relationships were also found in B. mori and $M$. sexta $[7,8]$. Since immulectins broadly participate in regulating the innate immune responses, the expansion of immulectins may greatly improve the survival rates of lepidopteran pests in the natural environment.

The expression profile of developmental stages shows that most IMLs express in larvae and pupae. Only three IMLs (IML-4, IML-5, and IML-18) express in adults. Interestingly, most CTL-S express in eggs and early-stage larvae (Figure 7A). These results suggest that CTL-S may be important for the development of and immunity in embryos and early stage larvae, while IMLs are critical for immunity in larvae, pupae, and adults. A Periplaneta lectin participates in the organization or stabilization of the epidermis during leg regeneration [49]. H. armigera CTL3 maintains normal larval growth and development by maintaining ecdysone and juvenile hormone signaling and suppressing the abundance of Enterocuccus mundtii [50]. CTLs also show a specific spatial expression pattern in naïve larval tissues: hemocytes, fat body, and epidermis are responsible for expressing CTLs (Figure 7B). These are the major larval tissues generating immune molecules. Bacterial and fungal infections induced dramatic changes in the expression of some CTLs (Figures 8 and 9). Our findings are similar to previous transcriptomic studies. In the cotton bollworm $H$. armigera, a transcriptome-based analysis showed that most CTL genes did not undergo any significant changes in the second instar larvae after $B$. bassiana infection, while most of them were upregulated in the fat body of the fifth instar larvae [17]. Although we did not compare the induction of CTLs between early stage and late-stage M. separata larvae, we found that most IMLs were expressed in mid-late-stage larvae and pupae. These data suggest that IMLs are important for immune responses in these stages. In the Japanese pine sawyer beetle, Monochamus alternatus infected with the entomopathogenic fungus Metarhizium anisopliae, several differentially expressed unigenes were CTLs [51]. Twelve CTL genes were identified in Adelphocoris suturalis (Hemiptera: Miridae) immune responsive genes against fungal and bacterial pathogens [52]. Fourteen CTLs were identified in immunity-related genes in Ostrinia furnacalis against entomopathogenic fungi [53].

A simple rule to predict the ligand specificity of CRDs is based on some key residues: 'EPN'-motif CRDs can recognize mannose-type ligands; 'QPD'-motif CRDs usually recognize galactose-type ligands. Mutating 'EPN' in MBP-A to 'QPD' caused a shift from mannose to galactose ligands [54]. Conversely, mutating 'QPD' in sea cucumber CEL-I to 'EPN' led to a weak binding affinity for mannose [55]. Some surrounding residues or structures also can affect ligand selection. An additional mutation of $\operatorname{Tr} \mathrm{p}^{105}$ to His in MBP-A further increased the affinity to mannose [55]. A glycine-rich loop helps to exclude 
mannose and accommodate galactose in Gal-type CTLs [56,57]. However, there are exceptions to this rule. CEL-IV, a CTL in sea cucumber, Cucumaria echinate, contains the 'EPN' motif but binds galactose [58]. TC14, a CTL from the tunicate Polyandrocarpa misakiensis contains 'EPS' but binds galactose [59]. Some CRDs bind carbohydrates in the absence of the 'EPN/QPD' motif or $\mathrm{Ca}^{2+}$. The CRD of eosinophil major basic protein (EMBP) binds to heparin and heparan sulfate at a different contact site through electrostatic interactions and hydrogen bonds [60]. Bivalve lectins SPL-1 and SPL-2, which contained 'RPD' and 'KPD' motifs, showed $\mathrm{Ca}^{2+}$-independent binding affinity for GlcNAc or GalNAc [61]. Structural studies have elucidated how these residues interact with $\mathrm{Ca}^{2+}$ and carbohydrates. For monosaccharide ligands, steric restrictions are imposed by the coordination bonds and hydrogen bonds formed between 3-OH/4-OH, $\mathrm{Ca}^{2+}$, and 'EPN/QPD' motifs. Mannose has equatorial 3-OH and equatorial 4- $-\mathrm{OH}$, while galactose has equatorial 3-OH and axial 4-OH. In addition, the hydrogen donors and acceptors are reversed between $\mathrm{E}$ (acceptor)$\mathrm{P}-\mathrm{N}$ (donor) and $\mathrm{Q}$ (donor)-P-D(acceptor) (Figure 6D,E). Therefore, the predicted ligand specificity needs to be verified experimentally.

To sum up, this study built a de novo transcriptome assembly of $M$. separata larvae, from which 6 'S-type' and 29 'IML-type' CTLs were identified. Sequence features, phylogenetic relationships, ligand specificity, and expression profiles were studied. Further studies are required to explore the function of each CTL in the oriental armyworm.

Supplementary Materials: The following are available online at https: / www.mdpi.com/article/ 10.3390/insects12060559/s1, Figure S1: Sequence alignment of three types of CRDs; Table S1: List of Primers.

Author Contributions: Conceptualization, X.J.R.; methodology, H.L.; investigation, H.L., F.-F.L., L.-Q.F., Z.L., W.-T.Z. and Q.W.; data curation, H.L. and X.-J.R.; writing-original draft preparation, H.L. and X.-J.R.; writing-review and editing, X.-J.R.; supervision, X.-J.R.; project administration, X.-J.R.; funding acquisition, X.-J.R. All authors have read and agreed to the published version of the manuscript.

Funding: This study was supported by grants from the National Natural Science Foundation of China (31872284) and the Natural Science Foundation of Anhui Province (2008085J17) to X.-J.R.

Institutional Review Board Statement: Not applicable.

Data Availability Statement: Not applicable.

Conflicts of Interest: The authors declare no conflict of interest.

\begin{abstract}
Abbreviations
PRR: pattern recognition receptor; PAMP: pathogen-associated molecular pattern; CTL: C-type lectin; PGRP: peptidoglycan-recognition protein; $\beta$ GRP: $\beta$-1,3-glucan recognition protein; CRD: carbohydrate-recognition domain; CTLD: C-type lectin-like domain; IML: immulectin; PBS, phosphatebuffered saline; RT-qPCR: quantitative reverse transcription PCR; SMART: Simple Modular Architecture Research Tool; I-TASSER: Iterative Threading Assembly Refinement.
\end{abstract}

\title{
References
}

1. Janeway, C.A., Jr.; Medzhitov, R. Innate immune recognition. Annu. Rev. Immunol. 2002, 20, 197-216. [CrossRef]

2. Lin, Z.; Wang, J.L.; Cheng, Y.; Wang, J.X.; Zou, Z. Pattern recognition receptors from lepidopteran insects and their biological functions. Dev. Comp. Immunol. 2020, 108, 103688. [CrossRef] [PubMed]

3. Imler, J.L. Overview of Drosophila immunity: A historical perspective. Dev. Comp. Immunol. 2014, 42, 3-15. [CrossRef]

4. Rao, X.J.; Zhan, M.Y.; Pan, Y.M.; Liu, S.; Yang, P.J.; Yang, L.L.; Yu, X.Q. Immune functions of insect $\beta$ GRPs and their potential application. Dev. Comp. Immunol. 2018, 83, 80-88. [CrossRef] [PubMed]

5. Xia, X.; You, M.; Rao, X.J.; Yu, X.Q. Insect C-type lectins in innate immunity. Dev. Comp. Immunol. 2018, 83, 70-79. [CrossRef]

6. Zelensky, A.N.; Gready, J.E. The C-type lectin-like domain superfamily. FEBS J. 2005, 272, 6179-6217. [CrossRef]

7. Rao, X.J.; Shahzad, T.; Liu, S.; Wu, P.; He, Y.T.; Sun, W.J.; Fan, X.Y.; Yang, Y.F.; Shi, Q.; Yu, X.Q. Identification of C-type lectin-domain proteins (CTLDPs) in silkworm Bombyx mori. Dev. Comp. Immunol. 2015, 53, 328-338. [CrossRef] 
8. Rao, X.J.; Cao, X.; He, Y.; Hu, Y.; Zhang, X.; Chen, Y.R.; Blissard, G.; Kanost, M.R.; Yu, X.Q.; Jiang, H. Structural features, evolutionary relationships, and transcriptional regulation of C-type lectin-domain proteins in Manduca sexta. Insect Biochem. Mol. Biol. 2015, 62, 75-85. [CrossRef]

9. Nagae, M.; Yamaguchi, Y. Structural Aspects of Carbohydrate Recognition Mechanisms of C-Type Lectins. In C-Type Lectins in Immune Homeostasis, 2019/11/30 ed.; Yamasaki, S., Ed.; Springer: Cham, Switzerland, 2019; Volume 429, pp. 147-176.

10. Weis, W.I.; Drickamer, K.; Hendrickson, W.A. Structure of a C-type mannose-binding protein complexed with an oligosaccharide. Nature 1992, 360, 127-134. [CrossRef]

11. Yu, X.Q.; Gan, H.; Kanost, M.R. Immulectin, an inducible C-type lectin from an insect, Manduca sexta, stimulates activation of plasma prophenol oxidase. Insect Biochem. Mol. Biol. 1999, 29, 585-597. [CrossRef]

12. Yu, X.Q.; Kanost, M.R. Immulectin-2, a lipopolysaccharide-specific lectin from an insect, Manduca sexta, is induced in response to gram-negative bacteria. J. Biol. Chem. 2000, 275, 37373-37381. [CrossRef] [PubMed]

13. Yu, X.Q.; Ling, E.; Tracy, M.E.; Zhu, Y. Immulectin-4 from the tobacco hornworm Manduca sexta binds to lipopolysaccharide and lipoteichoic acid. Insect Mol. Biol. 2006, 15, 119-128. [CrossRef] [PubMed]

14. Ao, J.; Ling, E.; Yu, X.Q. Drosophila C-type lectins enhance cellular encapsulation. Mol. Immunol. 2007, 44, 2541-2548. [CrossRef]

15. Bi, J.; Ning, M.; Li, J.; Zhang, P.; Wang, L.; Xu, S.; Zhong, Y.; Wang, Z.; Song, Q.; Li, B. A C-type lectin with dual-CRD from Tribolium castaneum is induced in response to bacterial challenge. Pest Manage. Sci. 2020, 76, 3965-3974. [CrossRef]

16. Wang, J.L.; Zhang, Q.; Tang, L.; Chen, L.; Liu, X.S.; Wang, Y.F. Involvement of a pattern recognition receptor C-type lectin 7 in enhancing cellular encapsulation and melanization due to its carboxyl-terminal CRD domain in the cotton bollworm, Helicoverpa armigera. Dev. Comp. Immunol. 2014, 44, 21-29. [CrossRef] [PubMed]

17. Cheng, Y.; Lin, Z.; Wang, J.M.; Xing, L.S.; Xiong, G.H.; Zou, Z. CTL14, a recognition receptor induced in late stage larvae, modulates anti-fungal immunity in cotton bollworm Helicoverpa armigera. Dev. Comp. Immunol. 2018, 84, 142-152. [CrossRef]

18. Koizumi, N.; Imamura, M.; Kadotani, T.; Yaoi, K.; Iwahana, H.; Sato, R. The lipopolysaccharide-binding protein participating in hemocyte nodule formation in the silkworm Bombyx mori is a novel member of the C-type lectin superfamily with two different tandem carbohydrate-recognition domains. FEBS Lett. 1999, 443, 139-143. [CrossRef]

19. Watanabe, A.; Miyazawa, S.; Kitami, M.; Tabunoki, H.; Ueda, K.; Sato, R. Characterization of a novel C-type lectin, Bombyx mori multibinding protein, from the B. mori hemolymph: Mechanism of wide-range microorganism recognition and role in immunity. J. Immunol. 2006, 177, 4594-4604. [CrossRef] [PubMed]

20. Fang, Q.; Wang, F.; Gatehouse, J.A.; Gatehouse, A.M.; Chen, X.X.; Hu, C.; Ye, G.Y. Venom of parasitoid, Pteromalus puparum, suppresses host, Pieris rapae, immune promotion by decreasing host C-type lectin gene expression. PLoS ONE 2011, 6, e26888 [CrossRef]

21. Cheng, G.; Cox, J.; Wang, P.; Krishnan, M.N.; Dai, J.; Qian, F.; Anderson, J.F.; Fikrig, E. A C-type lectin collaborates with a CD45 phosphatase homolog to facilitate West Nile virus infection of mosquitoes. Cell 2010, 142, 714-725. [CrossRef] [PubMed]

22. Jiang, X.; Luo, L.; Zhang, L.; Sappington, T.W.; Hu, Y. Regulation of migration in Mythimna separata (Walker) in China: A review integrating environmental, physiological, hormonal, genetic, and molecular factors. Environ. Entomol. 2011, 40, 516-533. [CrossRef]

23. Wang, C.; Wang, S. Insect Pathogenic Fungi: Genomics, Molecular Interactions, and Genetic Improvements. Annu. Rev. Entomol. 2017, 62, 73-90. [CrossRef] [PubMed]

24. Qu, S.; Wang, S.B. Interaction of entomopathogenic fungi with the host immune system. Dev. Comp. Immunol. 2018, 83, 96-103. [CrossRef] [PubMed]

25. Liu, F.F.; Li, H.; Yang, P.J.; Rao, X.J. Structure-function analysis of PGRP-S1 from the oriental armyworm, Mythimna separata. Arch Insect Biochem. Physiol. 2021, 106, e21763. [CrossRef]

26. Liu, F.F.; Ding, C.; Yang, L.L.; Li, H.; Rao, X.J. Identification and analysis of two lebocins in the oriental armyworm Mythimna separata. Dev. Comp. Immunol. 2021, 116, 103962. [CrossRef] [PubMed]

27. Yang, P.-J.; Zhan, M.-Y.; Yang, L.-L.; Liu, Q.-Q.; Xu, Y.; Pan, Y.-M.; Rao, X.-J. Characterization of PGRP-S1 from the oriental armyworm, Mythimna separata. Dev. Comp. Immunol. 2019, 90, 121-129. [CrossRef] [PubMed]

28. Martin, M. Cutadapt removes adapter sequences from high-throughput sequencing reads. EMBnet.journal 2011, 17, 3. [CrossRef]

29. Haas, B.J.; Papanicolaou, A.; Yassour, M.; Grabherr, M.; Blood, P.D.; Bowden, J.; Couger, M.B.; Eccles, D.; Li, B.; Lieber, M.; et al. De novo transcript sequence reconstruction from RNA-seq using the Trinity platform for reference generation and analysis. Nat. Protoc. 2013, 8, 1494-1512. [CrossRef]

30. Altschul, S.F.; Madden, T.L.; Schäffer, A.A.; Zhang, J.; Zhang, Z.; Miller, W.; Lipman, D.J. Gapped BLAST and PSI-BLAST: A new generation of protein database search programs. Nucleic Acids Res. 1997, 25, 3389-3402. [CrossRef]

31. Waterhouse, A.M.; Procter, J.B.; Martin, D.M.A.; Clamp, M.; Barton, G.J. Jalview Version 2-A multiple sequence alignment editor and analysis workbench. Bioinformatics 2009, 25, 1189-1191. [CrossRef] [PubMed]

32. Kumar, S.; Stecher, G.; Li, M.; Knyaz, C.; Tamura, K. MEGA X: Molecular Evolutionary Genetics Analysis across Computing Platforms. Mol. Biol. Evol. 2018, 35, 1547-1549. [CrossRef]

33. Roy, A.; Kucukural, A.; Zhang, Y. I-TASSER: A unified platform for automated protein structure and function prediction. Nat. Protoc. 2010, 5, 725-738. [CrossRef]

34. Pettersen, E.F.; Goddard, T.D.; Huang, C.C.; Couch, G.S.; Greenblatt, D.M.; Meng, E.C.; Ferrin, T.E. UCSF Chimera-A visualization system for exploratory research and analysis. J. Comput. Chem. 2004, 25, 1605-1612. [CrossRef] [PubMed] 
35. Crooks, G.E.; Hon, G.; Chandonia, J.M.; Brenner, S.E. WebLogo: A sequence logo generator. Genome Res. 2004, 14, 1188-1190. [CrossRef] [PubMed]

36. Chen, C.; Chen, H.; Zhang, Y.; Thomas, H.R.; Frank, M.H.; He, Y.; Xia, R. TBtools: An Integrative Toolkit Developed for Interactive Analyses of Big Biological Data. Mol. Plant 2020, 13, 1194-1202. [CrossRef] [PubMed]

37. Lu, Y.; Su, F.; Zhu, K.; Zhu, M.; Li, Q.; Hu, Q.; Zhang, J.; Zhang, R.; Yu, X.-Q. Comparative genomic analysis of C-type lectin-domain genes in seven holometabolous insect species. Insect Biochem. Mol. Biol. 2020, 126, 103451. [CrossRef]

38. Koizumi, N.; Imai, Y.; Morozumi, A.; Imamura, M.; Kadotani, T.; Yaoi, K.; Iwahana, H.; Sato, R. Lipopolysaccharide-binding protein of Bombyx mori participates in a hemocyte-mediated defense reaction against gram-negative bacteria. J. Insect Physiol. 1999, 45, 853-859. [CrossRef]

39. Stark, R.; Grzelak, M.; Hadfield, J. RNA sequencing: The teenage years. Nat. Rev. Genet. 2019, 20, 631-656. [CrossRef] [PubMed]

40. Liu, Y.; Qi, M.; Chi, Y.; Wuriyanghan, H. De Novo Assembly of the Transcriptome for Oriental Armyworm Mythimna separata (Lepidoptera: Noctuidae) and Analysis on Insecticide Resistance-Related Genes. J. Insect Sci. 2016, 16. [CrossRef] [PubMed]

41. Wang, J.D.; Wang, W.Z.; Wang, Y.R.; Gao, S.J.; Elzaki, M.; Wang, R.; Wang, R.; Wu, M. Response of detoxification and immune genes and of transcriptome expression in Mythimna separata following chlorantraniliprole exposure. Comp. Biochem. Physiol. Part D Genom. Proteom. 2018, 28, 90-98. [CrossRef]

42. Duan, Y.; Gong, Z.; Wu, R.; Miao, J.; Jiang, Y.; Li, T.; Wu, X.; Wu, Y. Transcriptome analysis of molecular mechanisms responsible for light-stress response in Mythimna separata (Walker). Sci. Rep. 2017, 7, 45188. [CrossRef] [PubMed]

43. Liu, Z.; Wang, X.; Lei, C.; Zhu, F. Sensory genes identification with head transcriptome of the migratory armyworm, Mythimna separata. Sci. Rep. 2017, 7, 46033. [CrossRef]

44. Chang, X.Q.; Nie, X.P.; Zhang, Z.; Zeng, F.F.; Lv, L.; Zhang, S.; Wang, M.Q. De novo analysis of the oriental armyworm Mythimna separata antennal transcriptome and expression patterns of odorant-binding proteins. Comp. Biochem. Physiol. Part D Genom. Proteom. 2017, 22, 120-130. [CrossRef] [PubMed]

45. He, Y.Q.; Feng, B.; Guo, Q.S.; Du, Y. Age influences the olfactory profiles of the migratory oriental armyworm mythimna separate at the molecular level. BMC Genom. 2017, 18, 32. [CrossRef]

46. Du, L.; Zhao, X.; Liang, X.; Gao, X.; Liu, Y.; Wang, G. Identification of candidate chemosensory genes in Mythimna separata by transcriptomic analysis. BMC Genom. 2018, 19, 518. [CrossRef]

47. Huang, Y.; Yin, H.; Zhu, Z.; Jiang, X.; Li, X.; Dong, Y.; Sheng, C.; Liao, M.; Cao, H. Expression and functional analysis of cytochrome P450 genes in the integument of the oriental armyworm, Mythimna separata (Walker). Pest Manage. Sci. 2021, 77, 577-587. [CrossRef]

48. Cummings, R.; McEver, R. C-Type Lectins. In Essentials of Glycobiology [Internet], 3rd ed.; Varki, A., Cummings, R.D., Esko, J.D., Stanley, P., Hart, G.W., Aebi, M., Darvill, A.G., Kinoshita, T., Packer, N.H., Prestegard, J.H., et al., Eds.; Cold Spring Harbor Laboratory Press: Cold Spring Harbor, NY, USA, 2017.

49. Kubo, T.; Kawasaki, K.; Natori, S. Transient appearance and localization of a 26-kDa lectin, a novel member of the Periplaneta lectin family, in regenerating cockroach leg. Dev. Biol. 1993, 156, 381-390. [CrossRef]

50. Wang, W.; Wang, G.; Zhuo, X.; Liu, Y.; Tang, L.; Liu, X.; Wang, J. C-type lectin-mediated microbial homeostasis is critical for Helicoverpa armigera larval growth and development. PLoS Pathog. 2020, 16, e1008901. [CrossRef]

51. Kim, J.C.; Lee, M.R.; Kim, S.; Park, S.E.; Lee, S.J.; Shin, T.Y.; Kim, W.J.; Kim, J. Transcriptome Analysis of the Japanese Pine Sawyer Beetle, Monochamus alternatus, Infected with the Entomopathogenic Fungus Metarhizium anisopliae JEF-197. J. Fungi 2021, 7. [CrossRef] [PubMed]

52. Ma, M.; Guo, L.; Tu, C.; Wang, A.; Xu, L.; Luo, J. Comparative Analysis of Adelphocoris suturalis Jakovlev (Hemiptera: Miridae) Immune Responses to Fungal and Bacterial Pathogens. Front. Physiol. 2021, 12. [CrossRef] [PubMed]

53. Shen, D.X.; Liu, Y.; Zhou, F.; Wang, G.R.; An, C.J. Identification of Immunity-Related Genes in Ostrinia furnacalis against Entomopathogenic Fungi by RNA-Seq Analysis. PLoS ONE 2014, 9. [CrossRef]

54. Drickamer, K. Engineering galactose-binding activity into a C-type mannose-binding protein. Nature 1992, 360, 183-186. [CrossRef]

55. Moriuchi, H.; Unno, H.; Goda, S.; Tateno, H.; Hirabayashi, J.; Hatakeyama, T. Mannose-recognition mutant of the galactose/Nacetylgalactosamine-specific C-type lectin CEL-I engineered by site-directed mutagenesis. Biochim. Biophys. Acta 2015, 1850, 1457-1465. [CrossRef] [PubMed]

56. Iobst, S.T.; Drickamer, K. Binding of sugar ligands to $\mathrm{Ca}(2+)$-dependent animal lectins. II. Generation of high-affinity galactose binding by site-directed mutagenesis. J. Biol. Chem. 1994, 269, 15512-15519. [CrossRef]

57. Kolatkar, A.R.; Weis, W.I. Structural basis of galactose recognition by C-type animal lectins. J. Biol. Chem. 1996, 271 , 6679-6685. [CrossRef] [PubMed]

58. Hatakeyama, T.; Kamiya, T.; Kusunoki, M.; Nakamura-Tsuruta, S.; Hirabayashi, J.; Goda, S.; Unno, H. Galactose recognition by a tetrameric C-type lectin, CEL-IV, containing the EPN carbohydrate recognition motif. J. Biol. Chem. 2011, 286, 10305-10315. [CrossRef]

59. Poget, S.F.; Legge, G.B.; Proctor, M.R.; Butler, P.J.; Bycroft, M.; Williams, R.L. The structure of a tunicate C-type lectin from Polyandrocarpa misakiensis complexed with D -galactose. J. Mol. Biol. 1999, 290, 867-879. [CrossRef] 
60. Swaminathan, G.J.; Myszka, D.G.; Katsamba, P.S.; Ohnuki, L.E.; Gleich, G.J.; Acharya, K.R. Eosinophil-granule major basic protein, a C-type lectin, binds heparin. Biochemistry 2005, 44, 14152-14158. [CrossRef] [PubMed]

61. Unno, H.; Itakura, S.; Higuchi, S.; Goda, S.; Yamaguchi, K.; Hatakeyama, T. Novel Ca ${ }^{2+}$-independent carbohydrate recognition of the C-type lectins, SPL-1 and SPL-2, from the bivalve Saxidomus purpuratus. Protein Sci. 2019, 28, 766-778. [CrossRef] [PubMed] 